\title{
The investigation of viscous and structural damping for piezoelectric energy harvesters using only time-domain voltage measurements
}

\author{
Majid Khazaee ${ }^{1}$, John E. Huber ${ }^{2}$, Lasse Rosendahl ${ }^{1}$, Alireza Rezaniakolaie ${ }^{* 1}$ \\ ${ }^{1}$ Department of Energy Technology, Aalborg University, Pontopidanstraede 111, 9220 Aalborg, Denmark. \\ ${ }^{2}$ Department of Engineering Science, University of Oxford, Parks Rd, Oxford OX1 3PJ, United Kingdom.
}

\section{Abstract}

Knowing the nature of damping in piezoelectric energy harvesters can lead to proper damping and electromechanical models and designing highly efficient harvesters with less damping. As an attempt toward a better understanding of damping in piezoelectric energy harvesters, this paper presents experimental results for structural and viscous air damping coefficients extracted directly from voltage measurements under shock-induced tests. Free-vibration excitations are analyzed using the modified Short-Term Fourier Transform and Resampling method. Seven cases are studied, namely Macro Fiber Composite with different substrate shims, different bonding layers, and with or without a tip mass. The damping coefficients can be reliably extracted using an up-chirp driving signal and analyzing the system's decay curve, without the need for full measurement of harmonic response over a wide frequency range. The results also indicate that the damping coefficient is not independent of the base excitation amplitude and can increase up to $30 \%$. The relative significance of viscous air damping and structural damping mechanisms is identified in each case. The dependency of viscous air damping on the base excitation amplitude is also evaluated. The experimental results highlight the significance of the bonding layer in structural damping, which can account for approximately $60 \%$ of the total damping. In the absence of a substrate shim and bonding layer, the main contribution to energy dissipation is viscous air damping. While an added tip mass increases the output power, it also escalates the viscous air damping to approximately $40 \%$ due to increased beam tip deflection.

Keywords: Piezoelectric, Energy Harvesting, Viscous damping, Structural Damping, Bonding Layer.

\section{Introduction}

The recovery of wasted energy using energy harvesting technologies is now widespread due to the global energy crisis, rising power demand, and environmental pollution [1], leading to alternative ways to power electronic devices. Nowadays, microelectronic devices consume significantly less energy, thanks to rapid science and technology advances, enabling battery replacement or battery

* Author. Tel.: (+45) 99409276

E-mail address: alr@et.aau.dk. 
recharging [2]. This will enable electronic devices to be installed in remote areas using available natural energies, avoiding pollution, labor cost, and replacement cost [3]. The direct piezoelectric effect [4] has been widely used for vibration energy harvesting (VEH) applications [5], aiming to generate low power for small electronic components, such as autonomous sensors [6], or for largescale power generation, such as smart pavement systems [7]. The essential vibrational parameters in piezoelectric VEH are the natural frequency, the damping ratio, and mode shapes. Piezoelectric energy harvesters (PEHs) generate maximum voltage output when deformed with a driving frequency close to their fundamental frequency [8]. In many practical cases, an added mass can control the fundamental frequency [8,9], enabling structural deformation to be considerably amplified at the working frequency [10]. Moreover, any source of energy dissipation, such as strain rate material damping or air resistance, reduces the generated power [11], and accordingly, efforts are made to keep the damping ratio as low as possible in harvester design [12]. Finally, mode shapes determine the mechanical strain pattern in the energy harvester (strain is proportional to the applied stress [13]) and consequently influence the electrical power output.

Although damping affects the power output of PEHs [14], there appears to be no comprehensive multilayer piezoelectric harvester, the damping coefficients of different layers are often considered constant [7]. While studies calculate damping coefficients from measured responses, there are considerable differences in their results. For instance, DuToit and Wardle [15] reported mechanical damping of $\zeta_{m}=1.78 \%$ for a PZT-5A bimorph from Piezo Systems, Inc. (T226-A4-503X), while Erturk and Inman [16] reported $\zeta_{m}=2.7 \%$ for the same bimorph with $12 \mathrm{~g}$ added tip mass, and Kim et al. [17] reported $\zeta_{m}=8.2 \%$ for the same bimorph with the same added tip mass.

Energy dissipation sources comprise air resistance, support loss, and structural damping [18]. Typically, a cantilever PEH comprises several layers, with a piezoelectric layer bonded to other layers. The bonding layer may become a source of structural damping. In a hybrid material comprising two low damping materials, the viscoelastic contact layer's damping effect can be substantial [19]. Zhou et al. [20] investigated viscoelastic damping materials. Khazaee et al. [21] showed that the bonding layer condition could significantly affect mechanical damping. Arafa and Baz [22] investigated the piezoelectric composites' structural damping and showed that the composite internal structure would change the internal loss factor. Nevertheless, in many PEH studies, energy dissipation is assumed to be purely due to viscous damping [15], while, for instance, the damping source in a piezoelectric harvester can be due to the hysteresis behavior [23] and also internal material frictions. In piezoelectric composites, the structural lay-up can amplify the material internal damping 
[24]. Khazaee et al. [25] employed a simple one-degree-of-freedom model to show how different proportions of structural and viscous damping could affect resonant electrical power estimation. In particular, they showed that the use of a purely viscous damping model could lead to inaccuracy. Cooley et al. [26] pointed out the importance of viscous and structural damping models, stating that experimental tests are required to clarify viscous and structural damping roles in each configuration.

For the damping parameter determination in PEHs, the simple peak decay [27], the peak bandwidth [28], curve fitting [29], and the subspace system identification (SSI) [30] methods can be used. In the simple peak decay method, the damping parameter is estimated from the logarithmic decrement observed in the free-vibration response [31]. Due to noise in a measured time-domain signal, the decrement analysis is often accompanied by uncertainties. Moreover, the simple peak decay can only extract the fundamental vibration mode damping, not that for any higher modes. In the peak bandwidth method, the damping is extracted from the bandwidth of the frequency response function. In this method, the harvester needs to be excited in a wide range of frequencies for forming the frequency response function, which will be very time consuming because of the numerous test tries. Classical curve fitting or SSI methods, such as a circle or line fitting, require multi-sensory data obtained from various experimental tests followed by complex numerical and optimization algorithms [32].

An inaccurate assumption about the damping mechanism contributions leads to inaccurate power output estimation. Therefore, there is a need for a systematic method to identify and measure the contributions of viscous and structural damping mechanisms. Both viscous damping [33] and structural damping [34] can be significant in each specific application due to the load conditions, boundary conditions, and material lay-up. Banks and Inman [35] showed a combination model of the viscous air and the structural internal friction damping would have the best agreement with experimental results. Nonetheless, clarifying the contributions of viscous and structural damping mechanisms is still an unmet goal. Potentially, the bonding layer, as an inseparable part of piezoelectric energy harvesters, can be a source of damping; yet its damping effect has not been studied. Approaches for the damping coefficient extraction require many experimental tests, multisensory data, or complex numerical and optimization algorithms. Thus, deriving an objective approach applied directly on the piezoelectric voltage measurements, capable of extracting modal parameters for different vibration modes, is an unmet goal. In this study, a modified Short-Term

31 Fourier Transform and Resampling (STFR) approach is proposed for the damping determination that 
1 harvester. The modified STFR is capable of extracting modal parameters for each mode of interest from one set of data.

By testing piezoelectric harvesters at various base acceleration amplitudes and with different beam structures, the viscous air damping and the structural damping are determined. This systematic way of investigating the damping is novel. Furthermore, the present study experimentally separates the damping effects of the viscous air damping, the bonding layer's structural damping, and the structural damping of the piezoelectric composite. Different bonding layers, as an essential structural part in PEHs, and their damped responses are analyzed. Besides, the effect of added tip mass on the damping is investigated. These novel research outcomes extend the current knowledge of damping mechanisms in PEHs and enable accurate damping modeling in them. The approach presented here can be applied to different PEHs, and the identified viscous and structural damping coefficients can be used for accurate PEH modeling using a recently developed finite element model [36].

In this study, experimental investigations are carried out to find structural and viscous damping contributions to a piezoelectric harvester in conditions with and without added tip mass, with different substrate shims, and with different bonding layers. Macro Fiber Composite (MFC), a flexible piezoelectric composite, is used as the active layer. As discussed in section 2, experimental tests are carried out using a cantilever PEH in series with a resistive load and measuring only the load voltage to track the vibration of the cantilever. Observations of the decay of free-vibrations are used for extracting the damping coefficient, as in some previous studies [37]. However, in contrast to previous energy harvesting studies, here, the modified STFR, as discussed in section 3, is used. Section 4 presents air damping and structural damping data extracted for different configurations. The excitation acceleration effect on air-damping is also investigated for the bending modes in configurations with a substrate shim and without.

Comparison of different configurations shows that the structural damping coefficient for the bonding layer can be readily distinguished from viscous air damping, such that both may be quantified. The structural damping due to the bonding layer seems to be a substantial fraction of the total damping. It is shown that the viscous air damping depends on the harvester tip deflection and increases with tip deflection. The results also illustrate how the STFR method enables reliable extraction of damping parameters for different modes from a single decay curve.

\section{Experimental setup and piezoelectric samples}

The test rigs for all the experimental measurements are shown in Fig. 1Error! Reference source not found.(a) and (b). An aluminum base plate is used to connect a B\&K LDS V201 shaker to the 
1 piezoelectric sample as well as providing a site for the accelerometer. The piezoelectric sample is 2 clamped to the base plate at one end using an aluminum clamp bar, tightened with two bolts. The 3 amplifier is controlled with a National Instruments NI 9263 module, which generates analog voltage 4 signals. A KEPCO BOP 100-10MG amplifier and B\&K LPA 100 amplifier are used to amplify the 5 signals and power the shaker. The accelerometer measures the applied base excitation acceleration.

6 Output wires from each electrode of the piezoelectric harvester are connected across a purely resistive 7 load of resistance $R$. Two Data Acquisition (DAQ) systems are employed for reading the sensor and 8 piezoelectric voltage outputs, as shown in Fig.Error! Reference source not found. 1(c). The first

9 DAQ system is an 8-channel National Instruments NI 9201 module for reading the voltage across the resistive load (which is also the voltage across the harvester) and the DC accelerometer output for a 11 two seconds duration with a sampling rate of $F_{s}=6 \mathrm{kHz}$. Both the NI 9263 and NI 9201 modules are 12 placed in an NI cDAQ 9172 chassis connected with a USB cable to the computer. Labview ${ }^{\mathrm{TM}}$ software 13 is utilized for generating the desired signal and for recording data. The second DAQ system, B\&K 143676 stand-alone LAN-XI DAQ, is used for recording the B\&K accelerometer and the force 15 transducer data. The BK Connect software directly controls this DAQ system.

(a)

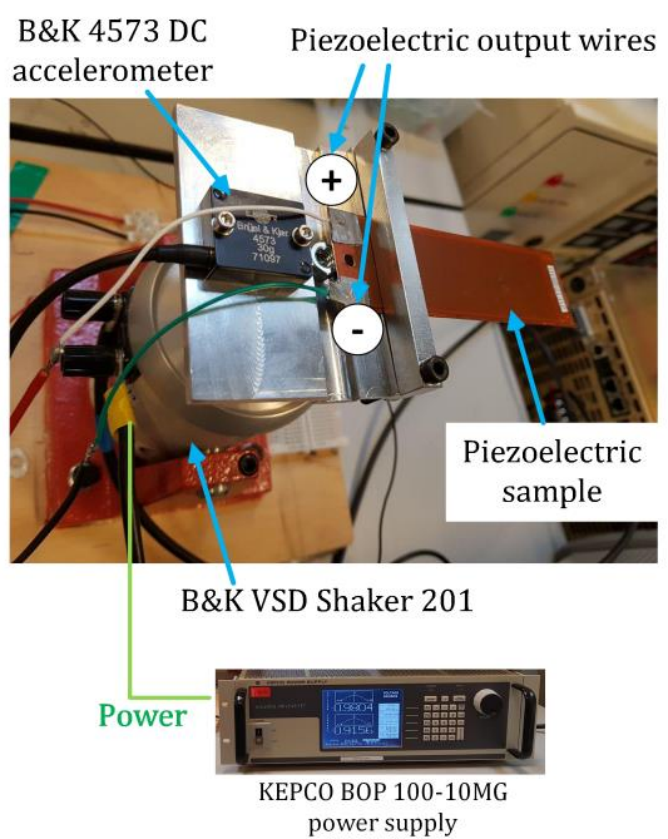

(b)

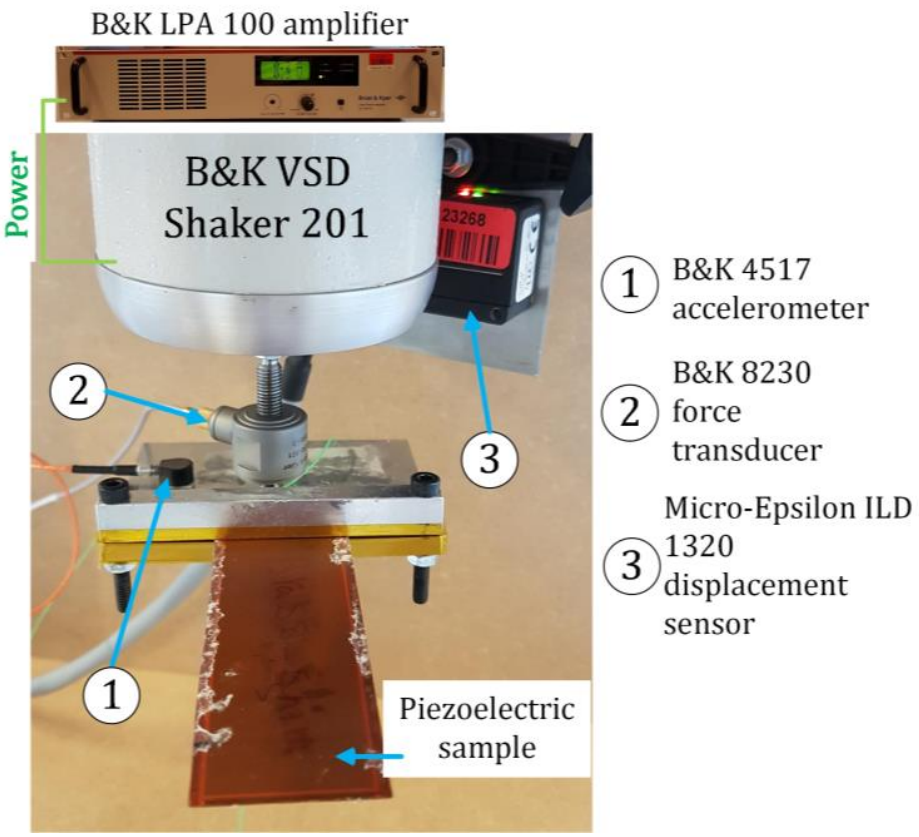




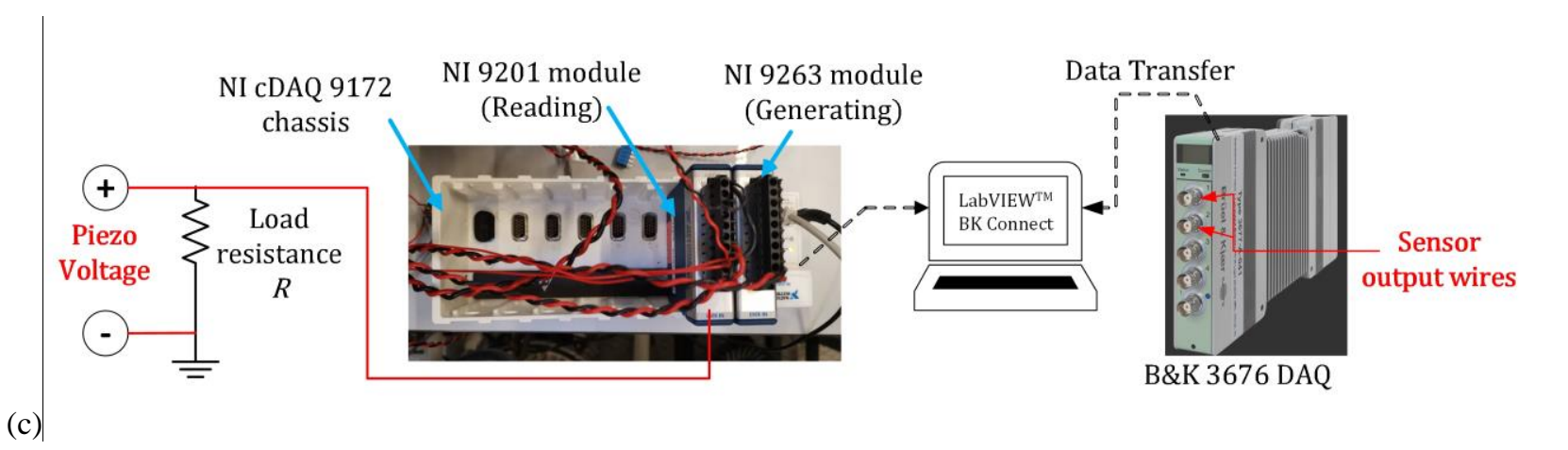

Fig. 1. (a) The piezoelectric sample with the cantilevered configuration attached to the shaker for Group I tests, (b) the cantilevered piezoelectric sample for Group II and III tests, and (c) data displacement laser sensor.

Seven samples are tested in this study; one comprises only the MFC of $0.3 \mathrm{~mm}$ thickness, while the others comprise the MFC, a substrate shim, and a bonding layer. The bonding layer joins the MFC and substrate shim. The added tip mass is also considered in some configurations. Error! Reference source not found. Fig. 2 (a) shows the sample with the substrate shim and the tip mass. The Macro Fiber Composite (MFC) used is M-8528-P2 type, from Smart Material Inc. [38], which serves as a piezoelectric bending energy harvester. The MFC has seven sub-layers: two Kapton outer layers, two acrylic layers, two electrodes, and one central active piezoelectric layer. More information about the MFC can be found in Smart Material Inc. [38].

Six different variations of the bonding layer and the substrate shim are tested. Loctite epoxy rapid 332, Loctite epoxy 3430, 3M Company 9084 double-sided tape, and Tesa ${ }^{\circledR} 4964$ double-sided adhesive tape are the four bonding layer materials. The $120-\mu \mathrm{m}$ aluminum, $100-\mu \mathrm{m}$ brass, and 100$\mu \mathrm{m}$ copper shims serve as substrates in the harvesters. Table 1 lists the harvester materials and the thickness properties. In some harvesters, a steel tip mass is added to the beam. In Group I tests, the tip mass is cylindrical, while in the group II and III tests, the tip mass is a rectangular steel shim. Fig. 2 (b) shows the tip mass for group II and III tests. To ease comparison of results, the samples are categorized into three groups, as shown in Table 1. Group I tests are designed to separate the viscous and structural damping and isolate the bonding layer damping effect. In Group II tests, a 60-mm length Tesa ${ }^{\circledR}$ adhesive tape is attached to the substrate shim, as shown in Fig. 2 (c), and the structural damping effect of this tape is studies. In Group III tests, different bonding layer types and their damping effects are investigated. The three types of bonding layers are illustrated in Fig. 2 (d)-(f). 
1 Stainless Steel, while the Tesa ${ }^{\circledR}$ adhesive tape is a $1200 \mu \mathrm{m}$ natural-rubber-type adhesive. The Tesa ${ }^{\circledR}$

2 adhesive tape is more elastic than the 3M Company's tape.

3 Table 1. The tested energy harvesters' properties, dimensions, and material structure include the piezoelectric, bonding, and substrate layers.

\begin{tabular}{|c|c|c|c|c|c|c|}
\hline \multirow[b]{2}{*}{$\begin{array}{l}\text { Group } \\
\text { No. }\end{array}$} & \multirow[b]{2}{*}{$\begin{array}{l}\text { Sample } \\
\text { No. }\end{array}$} & \multirow{2}{*}{$\begin{array}{l}\text { Piezoelectric } \\
\text { layer }\end{array}$} & \multirow[b]{2}{*}{ Substrate shim } & \multicolumn{2}{|c|}{ Bonding layer } & \multirow[b]{2}{*}{$\begin{array}{l}\text { Tip } \\
\text { mass (g) }\end{array}$} \\
\hline & & & & Material & $\begin{array}{c}\text { Thickness } \\
(\mu \mathrm{m})\end{array}$ & \\
\hline \multirow{2}{*}{ I } & 1 & MFC & - & - & - & $\begin{array}{l}0 \text { and } \\
4.2 \mathrm{~g}\end{array}$ \\
\hline & 2 & $t_{\mathrm{MFC}}=300 \mu \mathrm{m}$ & $t_{s}=120 \mu \mathrm{m}$ aluminum & Epoxy rapid 332 & $t_{c}=260$ & $\begin{array}{c}0 \text { and } \\
4.2 \mathrm{~g}\end{array}$ \\
\hline \multirow{2}{*}{ II } & 3 & MFC & $t_{s}=100 \mu \mathrm{m}$ brass & Epoxy 3430 & $t_{c}=300$ & $\begin{array}{l}0 \text { and } \\
9.2 \mathrm{~g}\end{array}$ \\
\hline & 4 & $t_{\mathrm{MFC}}=300 \mu \mathrm{m}$ & $\begin{array}{c}t_{s}=100 \mu \mathrm{m} \text { brass }+ \text { Tesa } \\
\text { adhesive tape }\end{array}$ & Epoxy 3430 & $t_{c}=300$ & $\begin{array}{l}0 \text { and } \\
9.2 \mathrm{~g}\end{array}$ \\
\hline \multirow{3}{*}{ III } & 5 & & $t_{s}=100 \mu \mathrm{m}$ copper & $\begin{array}{c}\text { 3M Company } \\
\text { double-sided tape }\end{array}$ & $t_{c}=100$ & - \\
\hline & 6 & $\begin{array}{c}\text { MFC } \\
t_{\mathrm{MFC}}=300 \mu \mathrm{m}\end{array}$ & $t_{s}=100 \mu \mathrm{m}$ copper & $\begin{array}{c}\text { 3M Company } \\
\text { double-sided tape }\end{array}$ & $t_{c}=300$ & - \\
\hline & 7 & & $t_{s}=100 \mu \mathrm{m}$ copper & Tesa $^{\circledR}$ adhesive tape & $t_{c}=1200$ & - \\
\hline
\end{tabular}

5

(a)

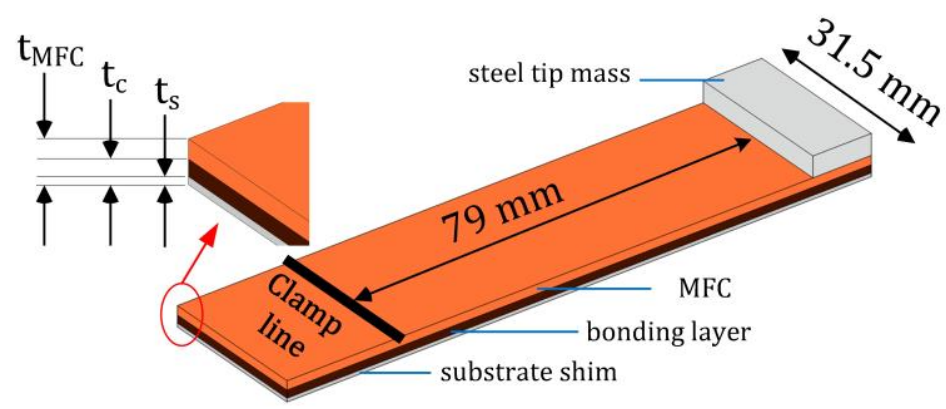

(b)

(c) 


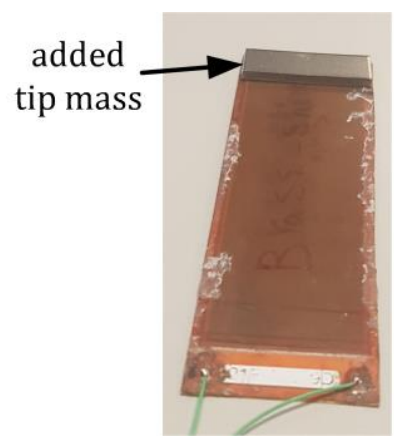

(d)

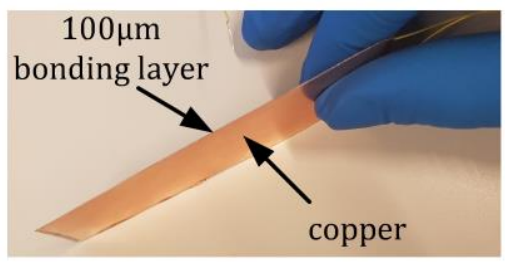

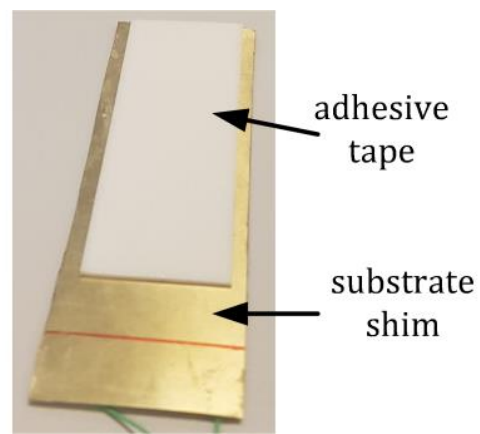

(e)

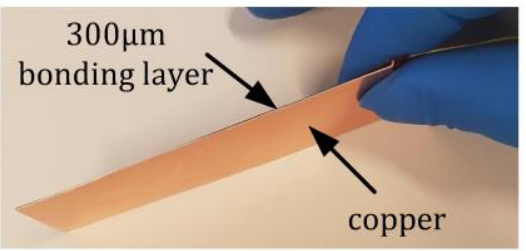

(f)

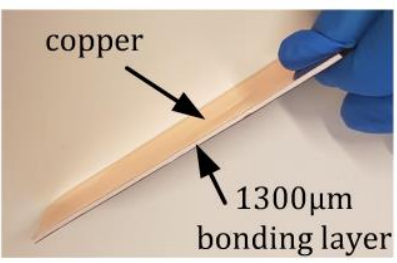

$$
f(t)=f_{0}+\frac{f_{1}-f_{0}}{T_{f}} t
$$

13 wherein $f_{0}$ is the initial frequency, $f_{1}$ is the target frequency, and $T_{f}$ is the target time. In this study, 14 parameters are set to $T_{f}=1 \mathrm{~s}, f_{0}=1 \mathrm{~Hz}$, and $f_{1}=1500 \mathrm{~Hz}$. Comparing a chirp signal to an impulse, their 15 power spectra are similar, but the phase spectra are distinct. The chirp signal excites a range of 
1 frequencies and is locally approximately periodic. The peak acceleration, $A_{c}=\left(2 \pi f_{1}\right)^{2} Y_{0}$, will be 2 used as a measure of the chirp acceleration amplitude. A section of the chirp signal used in this study, 3 along with its Fast Fourier Transform (FFT) and a typical system response, is shown in Fig. 3, where 4 the system's decaying free-vibration can be clearly seen.

(a)

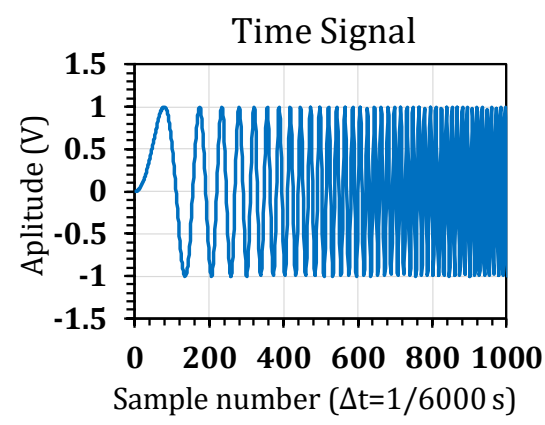

(b)

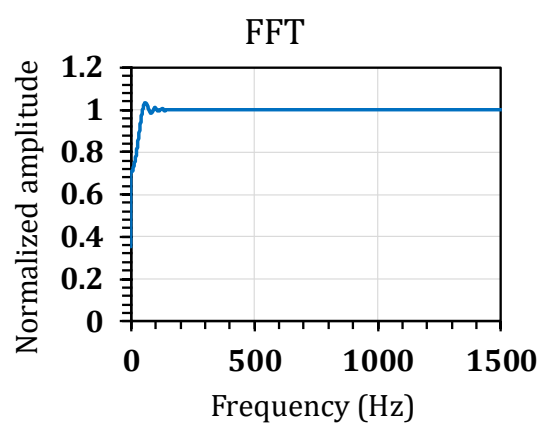

(c)

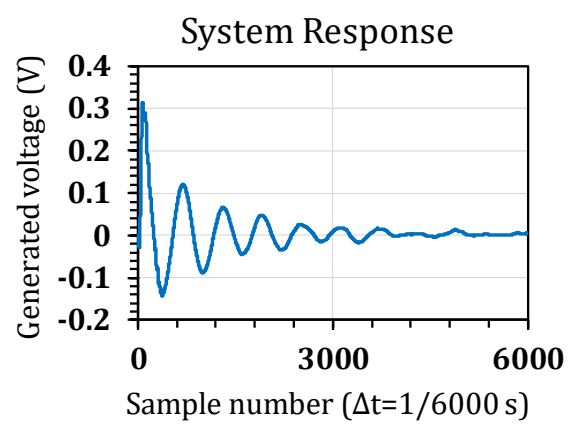

Fig. 3. (a) Typical examples of chirp excitation in the time domain (showing the first 1000 samples), (b) FFT of the input signal, and (c) system response, starting from the end of the chirp.

\section{Method for extracting resonant frequencies and damping coefficients}

This section presents a method for calculating natural frequencies and damping coefficients using only the measured output voltage response during the free vibration stage. The method relies on analyzing the decay curve; only the system response to the chirp signal (or any impulse-like input) is needed.

A method known as Short-Term Fourier transform and Resampling (STFR) has recently been developed for natural frequency and damping extraction in free vibration tests using the time response [39]. A series of modifications are applied to the method in order to increase its accuracy for this study.

First, the STFR is developed, and the relationship between the damping and the decay of the voltage response is illustrated using a recently measured voltage response of a piezoelectric harvester [40]. Because piezoelectricity is a transducing property giving a linear relationship between mechanical strain and electric field, the measured voltage characterizes the response of the system. The natural frequencies and damping ratios can be extracted from the voltage response without 21 requiring displacements or strains to be measured [16]. This simplifies the estimation of natural frequency and damping coefficient. Second, a Hann windowing function is introduced in the modified 
1 STFR for the segmenting of the output voltage signal; this smooths the segmented signals and

2 improves numerical stability. Third, a linear line fit on the logarithmic decay curve is introduced and compared with the exponential fit on the original STFR; this balances the contributions of the early peaks and the later peaks in the voltage response so that they contribute equally to the estimate of damping coefficient. Finally, in the presented STFR data, some optimization processes to maintain the curve fitting with the best possible accuracy are considered. These modifications will be described in detail in subsection 3.2.

\subsection{The damping coefficient in the decay voltage response}

Now consider how the damping coefficient can be found from the voltage response. Fig. 4 shows a measured free vibration response of sample 1 (No-Shim) harvester after an up-chirp base excitation.

11 It can be seen that the response has a maximum in the early stage and the voltage output then decays 12 away. Because the chirp excites a wide range of frequencies, the voltage response carries 13 superimposed characteristics from several vibration modes. A zoomed-in view of the free voltage 14 response in Fig. 4 shows high-frequency harmonics superimposed on the fundamental. As a result, modal characteristics from the higher frequency modes can be extracted from a single decay curve. The response for a system with well-separated modes is modeled as the summation of independent responses from $N$ vibration modes [41], and here we assume the same linear superposition feature. Moreover, from the general transient response of a single degree of freedom system, the voltage response for $i$-th mode can be expressed as [40]

$$
V^{i}[k]=A_{i} e^{-\zeta_{i} \bar{\omega}_{n, i}(k \Delta T)} \sin \left(\bar{\omega}_{d, i}(k \Delta T)+\theta_{i}\right)
$$

20 where $\bar{\omega}_{n, i}, \bar{\omega}_{d, i}$ and $\zeta_{i}$ are the undamped natural frequency, damped natural frequency, and 21 mechanical damping coefficient for the $i$-th mode. The damped natural frequency is given by $\bar{\omega}_{d, i}=\bar{\omega}_{n, i} \sqrt{1-\zeta_{i}^{2}}$. Furthermore, $A_{i}$ and $\theta_{i}$ are the amplitude and phase constants that depend upon 23 the initial conditions. The goal of the method in this study is to extract modal parameters, specifically $24 \bar{\omega}_{n_{i}}$ and $\zeta_{i}$. First, $\bar{\omega}_{d, i}$ is estimated, and then the decay curve is fitted to the functional form $A_{i} e^{-\zeta_{i} \bar{\omega}_{n, i}(k \Delta T)}$, enabling $\delta_{i}=\zeta_{i} \bar{\omega}_{n, i}$ to be evaluated. Having estimated $\delta_{i}$ and $\bar{\omega}_{d, i}$ for the mode under consideration, the damping coefficient can then be expressed as 


$$
\zeta_{i}=\frac{\delta_{i} / \bar{\omega}_{d, i}}{\sqrt{\left(1+\left(\delta_{i} / \bar{\omega}_{d, i}\right)^{2}\right)}}
$$

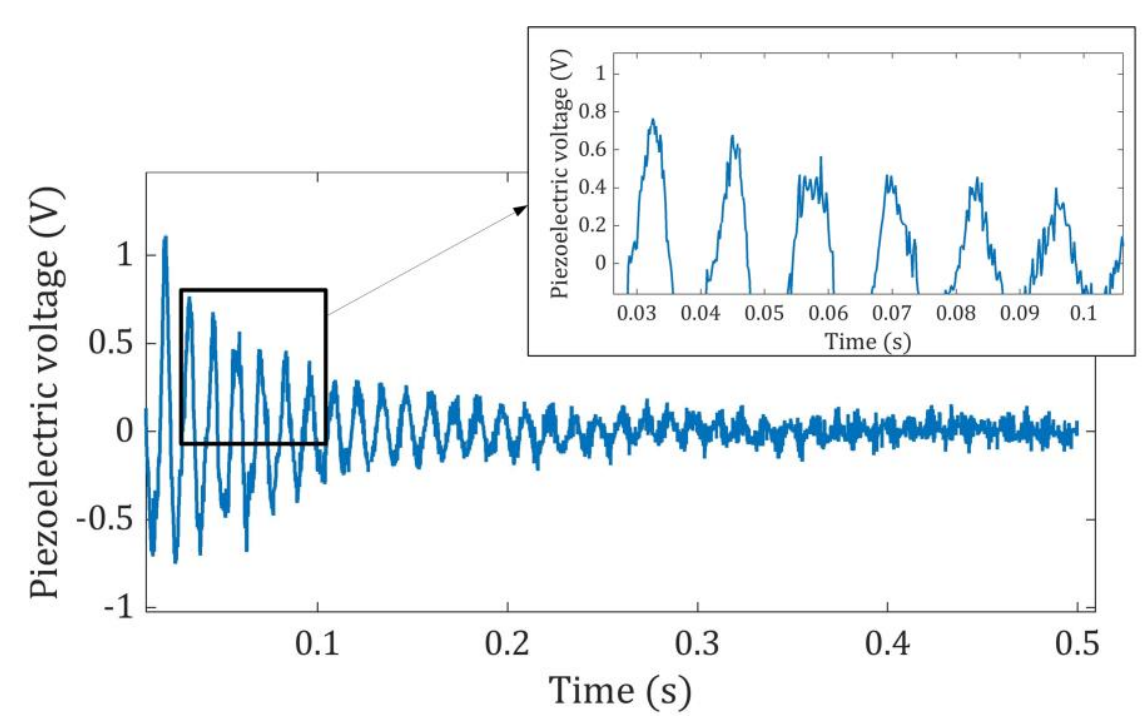

2 Fig. 4. A typical voltage response from a piezoelectric sample subjected to an up-chirp; the decay voltage response shows the effect of primary vibration mode and higher harmonics.

\section{$4 \quad 3.2$. Step-by-step guide for the STFR}

To extract the natural frequencies, the time response signal is divided into segments, and each segment is multiplied by a Hann function to perform smoothing at the cut-off edges. Next, each smoothed signal is transformed to the frequency domain using the Fast Fourier Transform (FFT). The results give a time-dependent frequency spectrum, the Short-Term Fourier Transform (STFT) with

9 Hann windowing. In order to extract $\delta_{i}$ for the $i$-th mode, the STFT is used to form a peak decay curve. This approach enables a separate curve fitting for each mode. Segmenting the signal reduces

11 the number of data points available in each FFT operation; to maintain resolution in frequency space,

12 each segment was first zero-padded and then resampled as described in detail below. Fig. 5 shows

13 the five main steps of the STFR method for extracting natural frequency and damping coefficients, 14 which are as follows: 
segmenting

(1) Segment and window signal
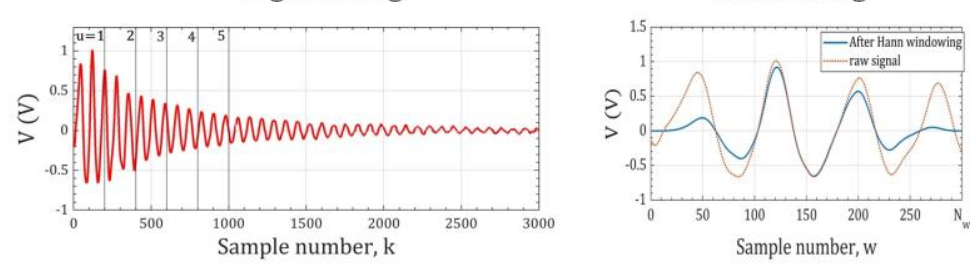

(2) Resample and zero-pad each segment

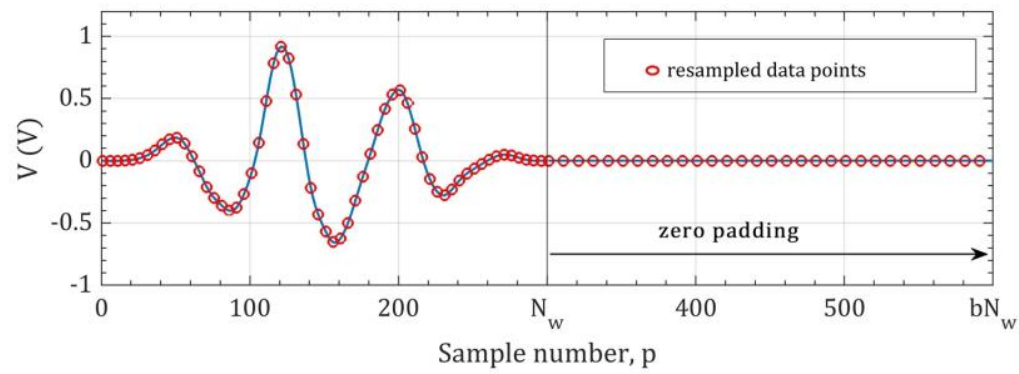

(3) STFT, extract peak

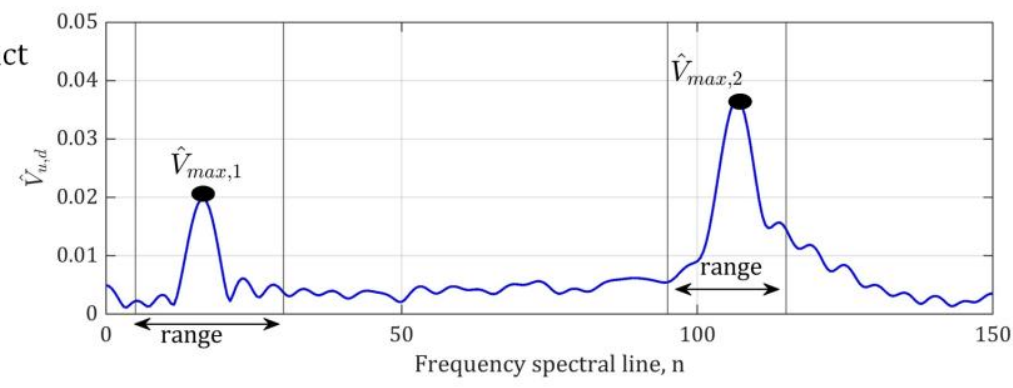

(4) Fit decay curve

(5) Extract modal parameters
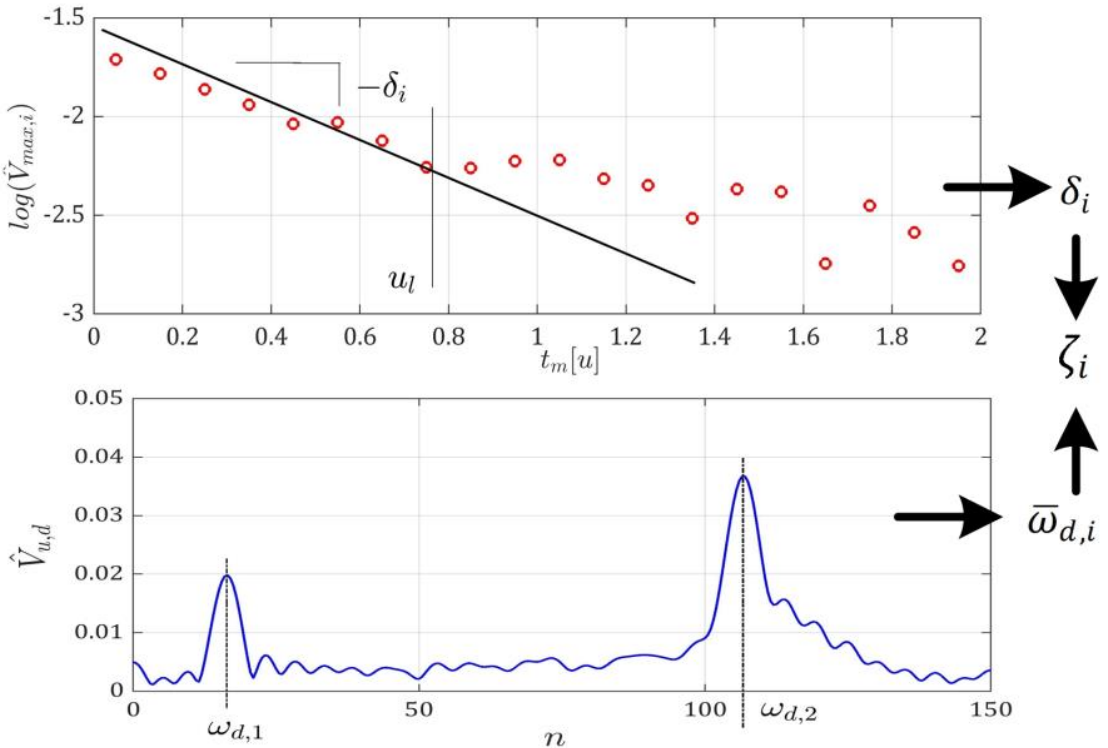

2 Fig. 5. Five main steps of the STFR process, (1) segmenting and windowing the voltage measured 3 signal, (2) resampling and zero-padding the segmented signal, (3) Applying FFT on the segmented 4 signal (STFT), (4) linear fit on the logarithm of the decay curve, (5) extracting the natural frequency 5 and damping coefficient.

6 Step 1 . Consider the voltage signal measured with a sampling rate $F_{s}$, with $N_{s}$ data points, specified 7 as a data sequence $V[k]$ : 


$$
V[k]=V(t=k \Delta T) \text { for } 0 \leq k \leq N_{s}-1
$$

1 where $t, \Delta T=1 / F_{S}$, and $k$ are, respectively, the time, time-step, and the sample number.

By assuming a linear superposition characteristic for the voltage signal, all the vibration modes' effect shall be seen in the voltage signal provided that their contribution to the voltage is significant.

4 In other words, the voltage signal is a summation of all the modes, $V[k]=\sum_{i=1}^{N} V^{i}[k]$.

The original measured signal $V[k]$ is divided into $U$ segments, and each divided signal is multiplied by a Hann function. The segmented signal is denoted by $V_{u}[w]\left(0 \leq w \leq N_{w}-1\right)$, where the number of data points in each segment is $N_{w}=N_{s} / U$. Each segment $0 \leq u \leq U-1$ can be expressed as

$$
V_{u}[w]=V\left(t=\left(u N_{w}+w\right) \Delta T\right) \mathcal{H}\left(w, N_{w}\right), \quad 0 \leq w \leq N_{w}-1 .
$$

9 where $w$ is an index number in each segment and $\mathcal{H}\left(w, N_{w}\right)$ is the symmetric Hann function with 10 length $N_{w}$. In the segmented data, the average time within each segment is given by

$$
t_{m}[u]=u N_{w} \Delta T+\frac{N_{w}}{2} \Delta T
$$

11 To generate the frequency spectrum from each segment of voltage measurements, the FFT is 12 applied to the segment, giving

$$
\widehat{V}_{u}[n]=\sum_{w=0}^{N_{w}-1}\left(V\left(t=\left(u N_{w}+w\right)\right) \Delta T\right) e^{-j 2 \pi \frac{n w}{N_{w}}}
$$

13 wherein $n$ is the spectral frequency line. The FFT frequency resolution equals to $\Delta \omega_{n, U}=\frac{2 \pi F_{s}}{N_{w}}=$ $14 \frac{2 \pi F_{S} U}{N_{S}}$. This shows that for a given total number of data points $\left(N_{S}\right)$, by increasing the number of 15 segments $(U), \Delta \omega_{n, U}$ increases, meaning that the accuracy of resonant frequency deteriorates.

Step 2. In order to improve the frequency resolution, the segmented signal is resampled by decimation with a factor $d$ and then is padded with zeroes by a factor of $b$. Thus, the total number of samples in the segmented signal is $N_{b}=b N_{w}$ and the sampling rate $F_{s}$ reduces to $F_{s} / d$. With this resampling and zero-padding, the frequency resolution becomes $\Delta \omega_{n, d}=\frac{2 \pi F_{s} U}{b d N_{s}}$. It is also worth mentioning that the Nyquist-Shannon sampling theorem should be satisfied when the decimation 
1 factor $d$ is being set in order to avoid aliasing. In this study, for instance, $U=40$, and $d=10$ is 2 employed.

3 Applying the FFT to the segment with resampling and zero-padding gives the spectrum

$$
\widehat{V}_{u, d}[n]=\sum_{p=0}^{N_{b}-1}\left(V\left(t=\left(u N_{w}+w\right)\right) d \Delta T\right) e^{-j 2 \pi \frac{n p}{N_{b}}} .
$$

4 Because the sampled data points in the segment are changed, the FFT peak value also changes, 5 and therefore a scaling is performed to up-scale $\widehat{V}_{u, d}[n]$ to that for the FFT without zero-padding, e.g. $6 \quad \widehat{V}_{u}[n]$.

7 Step 3. For each mode, a range of frequency is defined within which the natural frequency is expected to lie. Then, the FFT, $\widehat{V}_{u, d}[n]$, of each resampled segment is analyzed to find the maximum 9 value within the range, $\widehat{V}_{\max , i}[u]$.

10 Step 4. Plotting the peak values $\widehat{V}_{\max , i}[u]$ as a function of time forms a decay curve. Since the 11 decay curve for the $i$-th mode is expected to have the exponential form $A_{i} e^{-\zeta_{i} \bar{\omega}_{n, i}(k \Delta T)}$, the peak 12 values are represented on a logarithmic scale; there is then an approximately linear relationship 13 between segment number $u$ and the peak value logarithm. This gives all the data points the same 14 weight in a least-square straight line fit. From Eq. (3), for the $i$-th mode, a linear relationship between 15 the peak values of the STFT signals over time is expected, with

$$
\log \left(\widehat{V}_{\text {max }, i}[u]\right)=\log A_{i}-\delta_{i} t_{m}[u]
$$

16 where $-\delta_{i}$ is the slope of the logarithmic peak-value line obtained from the STFR process.

Step 5. The modal parameters $\bar{\omega}_{d, i}$ and $\zeta_{i}$ are extracted as follows: From each STFR segment, the damped natural frequency $\omega_{d, i}[u]$ is identified as the location of the peak value $\widehat{V}_{\max , i}[u]$. The average of the $\omega_{d, i}[u]$ values is used as an estimate of the damped natural frequency for the $i$-th mode, given by

$$
\bar{\omega}_{d, i}=\frac{1}{u_{l}} \sum_{u=0}^{u_{l}-1} \omega_{d, i}[u]
$$

21 Finally, $\zeta_{i}$ is obtained from $\bar{\omega}_{d, i}$ and $\delta_{i}$ using Eq. (4). 
In practice, steps 1 to 4 were repeated for different $U$ values in order to identify an optimum segment length. Choosing a large $U$ value produces a greater number of data points for line fitting by Eq.(10), but also reduces the number of data points per segment, $N_{w}$, which can result in aliasing. Typically, $U$ values of 20-40 were used. In addition, the $\widehat{V}_{\max , i}[u]$ data points eventually deviate from a linear regression at high values of $u$. This happens because the signal to noise ratio decreases as the signal decays [32]. Therefore, for calculating the damping coefficients from voltage measurements, the noise ratio is considered a data truncation factor. The data was truncated at a $u$ value, $u_{l}$, chosen to ensure that the error in the estimation of $\delta_{i}$ was less than $5 \%$ and the signal to noise ratio was greater than 5. The optimum truncation limit $u_{l}$ varies from test to test. In some cases, the first data point of the first segment exhibited an impact transient, which adversely affected curve fitting. In

11 these cases, the very first data point was excluded.

\subsection{Methodology for distinguishing the structural and viscous damping contributions}

The mechanical damping, $\zeta_{m}$, comprises a structural part, $\zeta_{\text {struc }}$, and an air (or viscous) damping part, $\zeta_{a}$. This damping representation demonstrates good agreement with experimental data [35]. According to two experimental studies by Woolam [42] and Baker et al. [43], material damping shows either no dependence or slight dependence on the amplitude of vibration. Therefore, the observed amplitude-dependency of $\zeta_{m}$ is due to amplitude-dependent increase in air damping. Baker et al. [43] showed experimentally that air damping varies approximately linearly with beam-tip deflection. Therefore, a combined damping model is considered as:

$$
\zeta_{m}=\zeta_{\text {struc }}+\alpha \zeta_{a}
$$

The STFR method calculates the damping at each shock-induced base excitation from the voltage, as shown in Fig. 6 (a). The damping model assumes that the air damping will also change due to changing beam tip deflection. Each sample is tested over a range of excitation magnitude to distinguish the structural and air damping. As the tip deflection increases, so also the viscous air damping increases. A line fit to the experimental damping coefficient data can be extrapolated back to the zero amplitude point to determine the structural damping contribution, see Fig. 6 (b). 
(a)

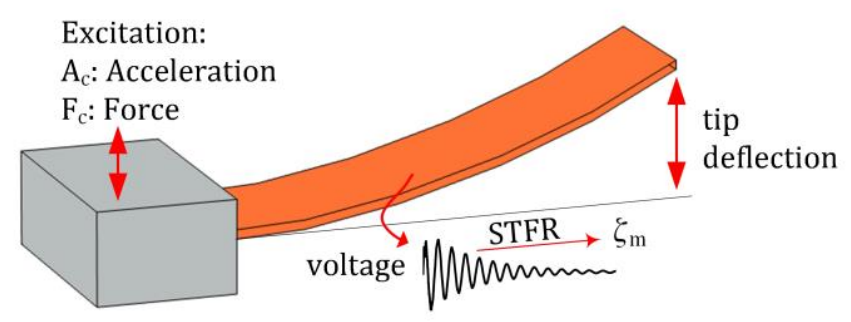

(b)

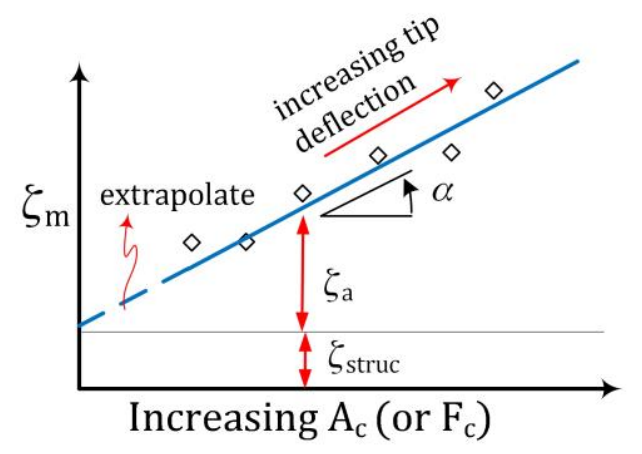

1 Fig. 6. (a) the deformed beam under a base excitation and the STFR method for extracting the damping coefficient, and (b) the process to distinguish the viscous and structural damping contributions in the total damping.

4 The process is applied to each sample for determining the structural damping effect of different 5 materials, the amplitude-dependent part of the damping, and the investigation of the added tip mass 6 effect on the damping. Fig. 7 presents the objectives of the experimental tests on the seven tested 7 samples. By comparing the data from different samples, materials, and lay-ups, the relative structural 8 and viscous damping contributions are studied. 


\begin{tabular}{|c|c|}
\hline sample 1 & $\begin{array}{l}\text { - Structural damping } \Rightarrow \text { for MFC } \\
\text { - Viscous damping } \Rightarrow \alpha \text { and effect of } 4.2 \mathrm{~g} \text { tip mass }\end{array}$ \\
\hline sample 2 & $\begin{array}{l}\text { - Structural damping } \Rightarrow \text { for epoxy bonding layer } \\
\text { - Viscous damping } \Rightarrow \alpha \text { and effect of } 4.2 \mathrm{~g} \text { tip mass }\end{array}$ \\
\hline sample 3 & $\begin{array}{l}\text { - Structural damping } \Rightarrow \text { for epoxy bonding layer } \\
\text { - Viscous damping } \Rightarrow \text { effect of } 9.2 \mathrm{~g} \text { tip mass }\end{array}$ \\
\hline sample 4 & $\begin{array}{l}\text { - Structural damping } \Rightarrow \text { for adhesive damping tape } \\
\text { - Viscous damping } \Rightarrow \text { effect of } 9.2 \mathrm{~g} \text { tip mass }\end{array}$ \\
\hline sample 5 & $\begin{array}{l}\text { - Structural damping } \Rightarrow \text { for the bonding layer of } 3 \mathrm{M} \\
100 \mu \mathrm{m} \text { double-sided tape }\end{array}$ \\
\hline sample 6 & $\begin{array}{l}\text { - Structural damping } \Rightarrow \text { for the bonding layer of } \\
300 \mu \mathrm{m} \text { double-sided tape }\end{array}$ \\
\hline sample 7 & $\begin{array}{l}\text { - Structural damping } \Rightarrow \text { for the bonding layer of } \\
\text { Tesa }^{\circledR} 1200 \mu \mathrm{m} \text { double-sided rubber-type tape }\end{array}$ \\
\hline
\end{tabular}

Fig. 7. The present study objectives for seven samples concerning the structural and viscous air damping parameters.

\section{Experimental Results}

In this section, the modal parameters extracted using the STFR approach from seven sample types are reported. Results are reported in three groups; each aims to emphasize one or two particular objectives. Group I and II are designed to distinguish the structural and viscous damping and their contribution to the total damping. The effect of added tip mass is also investigated in these group tests. In Group III tests, the main objective is to explore different bonding layers.

11 The electromechanical equations for a piezoelectric energy harvester are coupled equations relating mechanical vibration and electrical circuit parameters. In this work, the electrical load was optimized for maximum power output. For electrical load values close to the optimum load, the power output is insensitive to the electrical load [44] in an impact harvester, and therefore, it is not critical to precisely match the optimum load. Moreover, Erturk et al. [16] showed that the resonant frequency in short-circuit differs from the resonant frequency with a matched load by less than $0.5 \%$. Hence, it is accurate to infer the harvester's natural frequencies from the resonant frequencies extracted with the matched load present. The optimum load resistance was found to be $27 \mathrm{k} \Omega$ for all the harvesters used in this study. 
In order to test whether the choice of load resistor affects the damping parameters, decay curves were measured with a range of resistive loads: $R=100 \Omega, 27 \mathrm{k} \Omega$, and $10 \mathrm{M} \Omega$. A half-sine-impulse force was applied as the base excitation, and the resulting normalized voltage output is shown in Fig. 8. Comparing the voltage responses at $100 \Omega$ (similar to short-circuit condition) and $27 \mathrm{k} \Omega$ shows that they are almost identical, while for the $10 \mathrm{M} \Omega$ case (similar to open-circuit), a deviation in both frequency and decay rate is evident. Thus the modal parameters measured with the $27 \mathrm{k} \Omega$ optimum load in place are expected to match the short-circuit modal parameters.

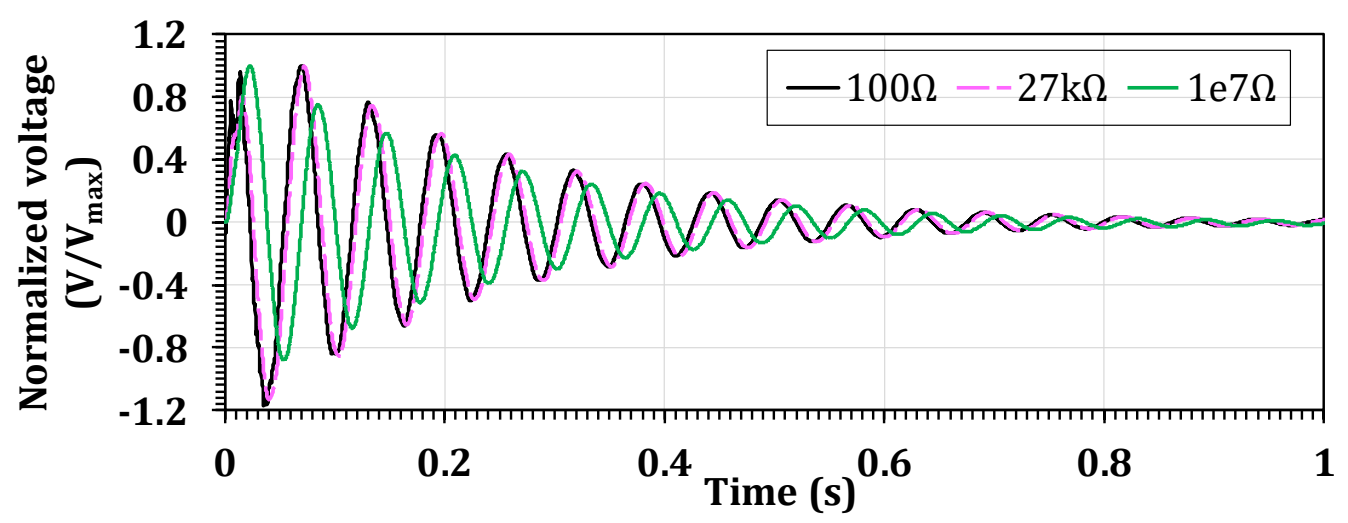

Fig. 8. Comparison of piezoelectric voltage outputs subjected to half-sine-impulse at different electrical loads.

\subsection{Group I tests (the MFC without and with the aluminum substrate)}

The Group I tests aim to separate viscous and structural damping roles in a cantilevered piezoelectric harvester in standard configuration and with a tip mass. Throughout this subsection, the label "No-Shim" refers to the MFC sample without substrate shim (sample 1), while "Alu-Shim" refers to the sample with a bonding layer and aluminum substrate shim (sample 2).

\subsubsection{Energy harvesters without tip mass}

Fig. 9 shows measured voltages from harvesters with and without substrate shim for the first $0.5 \mathrm{~s}$ after applying chirp excitation with a maximum acceleration of $1.77 \mathrm{~g}_{0}\left(g_{0}=9.81 \mathrm{~ms}^{-2}\right)$. Note that the Alu-Shim case generates considerably higher voltages compared to the No-Shim case. This shows that the substrate shim can positively affect power generation from an energy harvesting perspective despite losses in the bonding layer. Another interesting outcome can be seen in the zoomed view of the signals in Fig. 9. In the No-Shim sample, high frequency (>100 Hz) voltage variations can easily be seen, unlike the Alu-Shim voltage response. This can be linked to the behavior of the bonding layer in the Alu-Shim sample, which strongly damps high frequencies in the voltage response. 


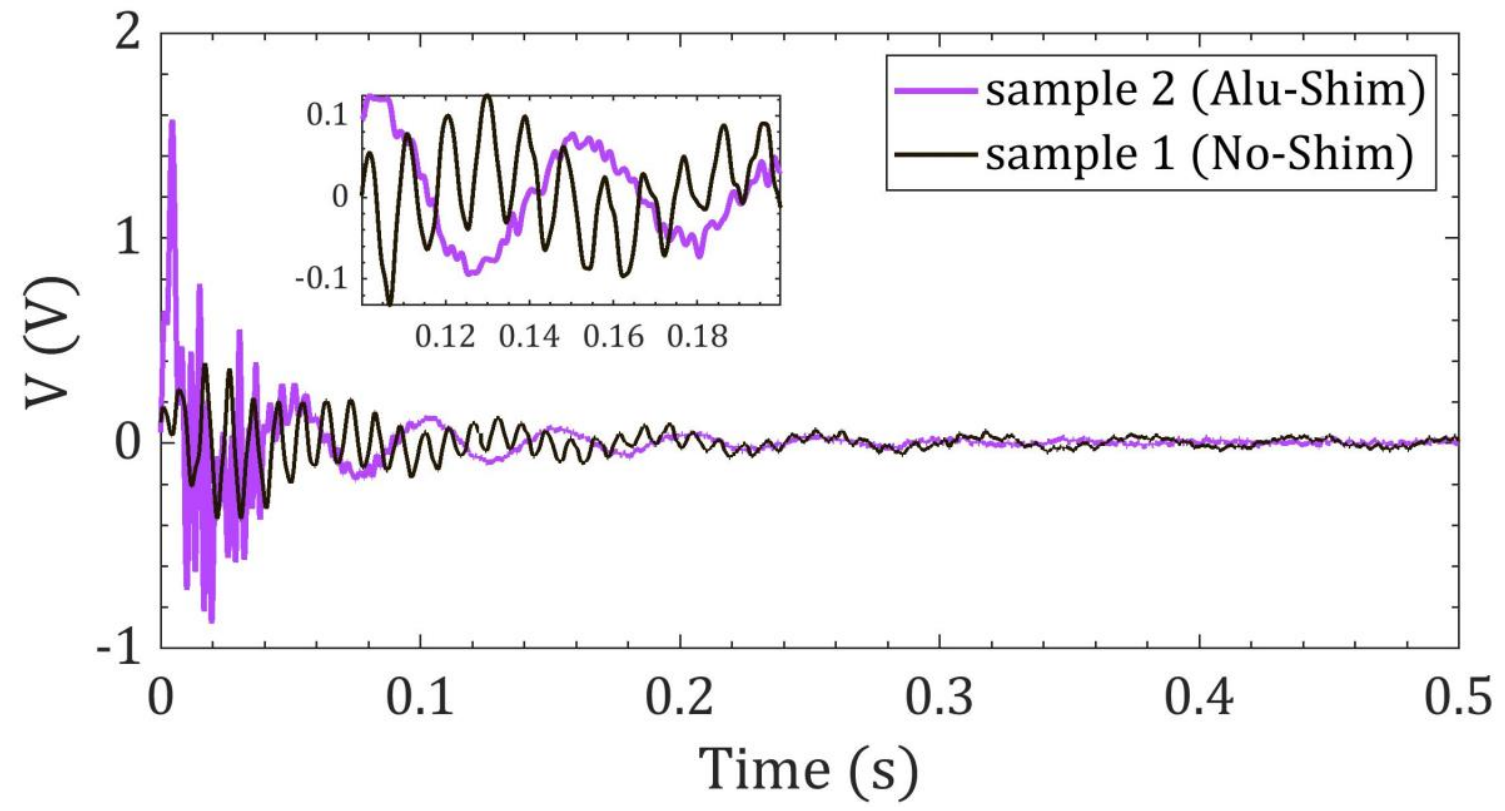

2 Fig. 9. Piezoelectric voltage outputs for sample 1 (No-Shim) and sample 2 (Alu-Shim) at $1.77 \mathrm{~g}_{0}$ chirp excitation amplitude with $R=27 \mathrm{k} \Omega$.

As a preliminary illustration of the resonant behavior of the energy harvesters, the FFT was applied to voltage responses measured after chirp excitation with varying acceleration. Fig. 10 shows that the

6 No-Shim sample exhibits two clearly defined resonances, while in the Alu-Shim sample, only the 7 first mode resonance is clearly visible. The first and second resonant frequencies for the No-Shim 8 sample are $17 \mathrm{~Hz}$ and $106 \mathrm{~Hz}$, respectively. Meanwhile, the first resonant frequency is approximately $920 \mathrm{~Hz}$ for the Alu-Shim sample. Another local maximum is at approximately $38 \mathrm{~Hz}$ due to anti10 resonance [45], similar to the observation in Khazaee et al. [46]. The natural frequencies and the 11 corresponding modes of the harvester were estimated using the finite element method by Khazaee et 12 al. [36]. This showed a torsional mode around $160 \mathrm{~Hz}$ and a bending mode around $190 \mathrm{~Hz}$ in the Alu13 Shim case; the corresponding resonant peaks can be seen in Fig. 10 (b).

(a) 

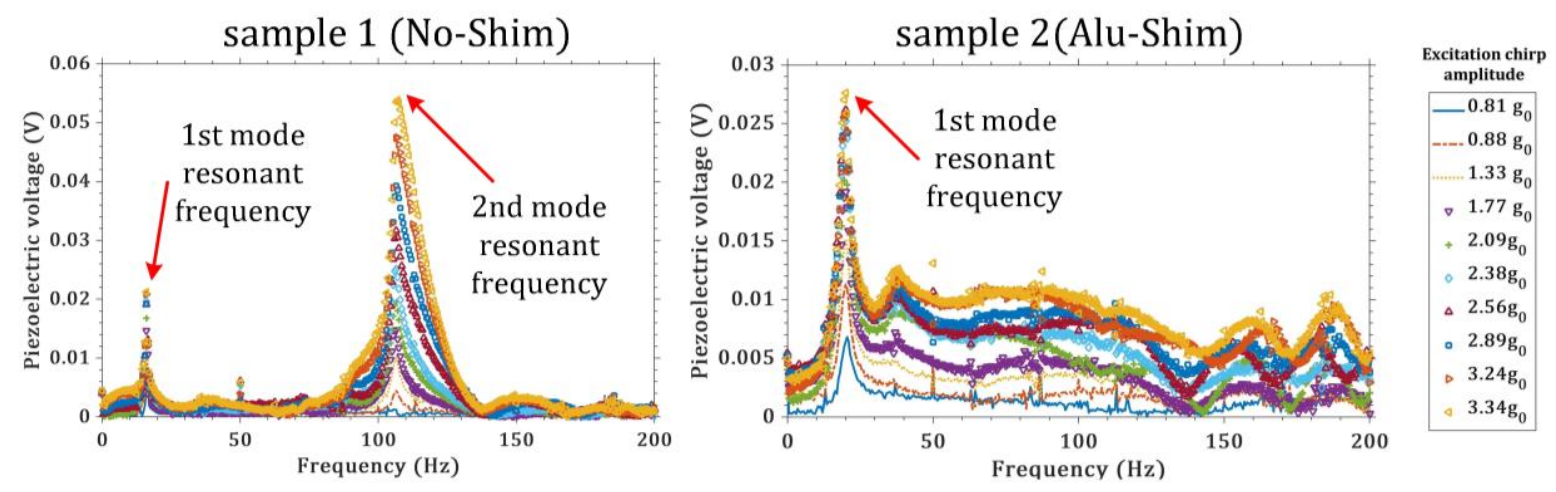

Fig. 10. Effect of increasing base chirp amplitude on STFT voltage responses for (a) sample 1 (No-Shim) and (b) sample 2 (Alu-Shim).

Next, the STFR approach is applied to free-vibration voltage responses in order to extract the resonant frequencies and damping ratios. Fig. 11 shows how the resonant frequency changes with 3 respect to chirp acceleration amplitude $\left(A_{c}\right)$ for both No-Shim and Alu-Shim samples. The first mode 4 resonant frequency of the Alu-Shim sample is approximately 20\% greater than that of the No-Shim 5 sample. The first resonant frequency of the No-Shim sample reduces as chirp acceleration increases, 6 perhaps due to amplitude dependence of the MFC layer stiffness. Khazaee et al. [47] have previously observed similar softening effects in an energy harvester. Nonetheless, the other natural frequencies shown in Fig. 11 are relatively independent of chirp acceleration.

(a)

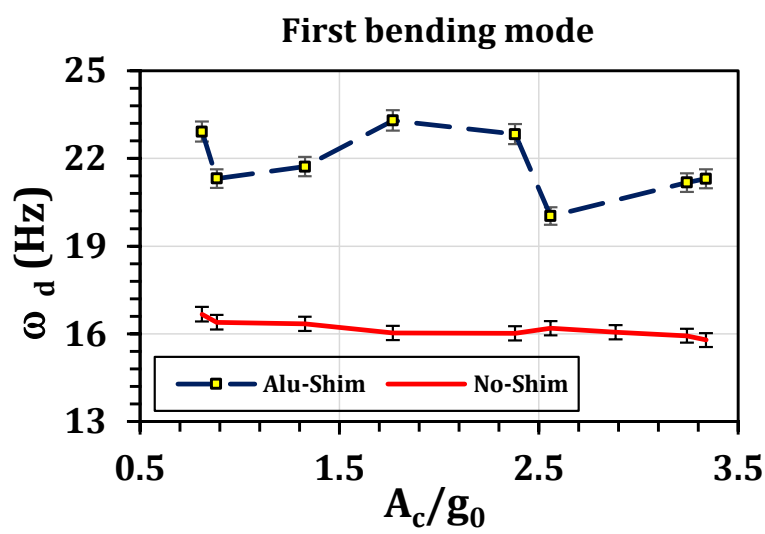

(b)

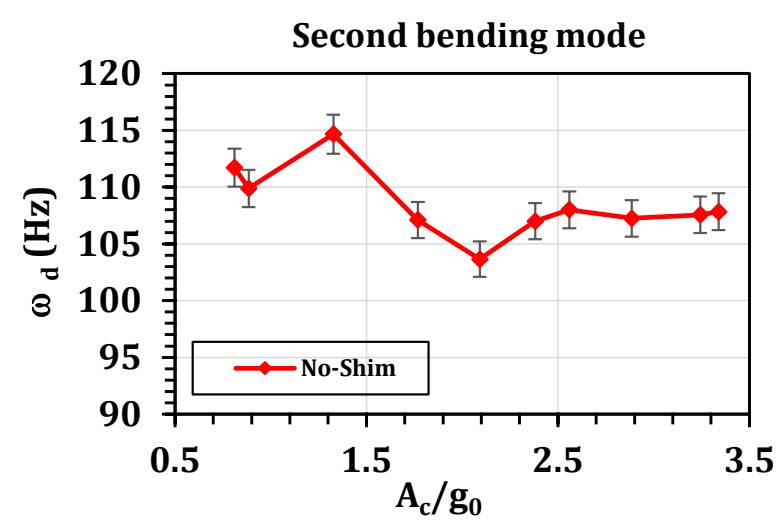

Fig. 11. Variation of resonant frequencies with respect to the base excitation amplitude (a) first bending mode for No-Shim and Alu-Shim samples, and (b) second bending mode for No-Shim sample.

The curve fitting results of STFR are used to extract mechanical damping, $\zeta_{m}$, for the Alu-Shim and No-Shim samples. The resulting $\zeta_{m}$ values for two bending modes in the No-Shim sample and 
1 the first bending mode in the Alu-Shim sample are shown in Fig. 12, as a function of chirp acceleration $A_{c}$. By comparing the damping coefficients in Fig. 12, mechanical damping increases monotonically with amplitude in an approximately linear way. Greater $A_{c}$ values lead to increased beam-tip deflection, and this, according to Woolam [42], increases mechanical damping. Similarly, as shown in Fig. 10, the damping has a linear dependency on base chirp acceleration, hence on beamtip deflection, assuming linearity.

The structural damping $\zeta_{\text {struc }}$ can also be evaluated from Fig. 12 , extrapolating to $A_{c}=0$. This indicates similar $\zeta_{\text {struc }}$ values for both modes in the No-Shim sample, but a much greater $\zeta_{\text {struc }}$ value for the Alu-Shim sample. Crandall [48] showed that adding a strip of viscoelastic damping tape to an aluminum plate can substantially increase the structural damping. An equivalent role is played here by the epoxy bonding layer in the Alu-Shim sample. The contribution of the aluminum shim to the structural damping is expected to be small [48].

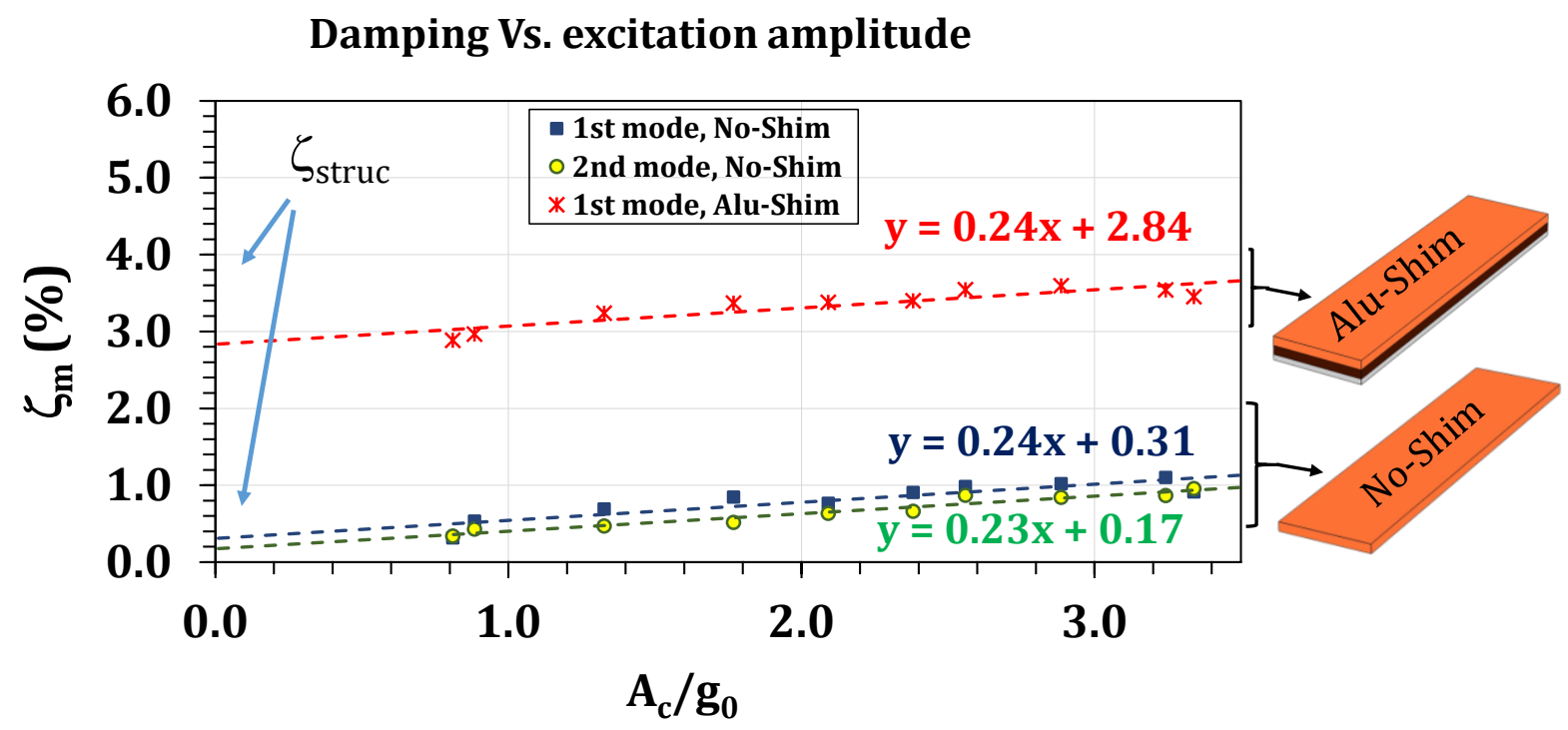

Fig. 12. Mechanical damping versus chirp base acceleration for the piezoelectric sample 1 (NoShim) and sample 2 (Alu-Shim).

\subsubsection{Effect of Added Tip Mass}

Tests were carried out with an added tip mass in the form of a $4.2 \mathrm{~g}$ cylindrical mass with $10 \mathrm{~mm}$ diameter and $4 \mathrm{~mm}$ height affixed to the upper surface of each harvester, with its centre $5 \mathrm{~mm}$ from the free end. Note that the No-Shim and Alu-Shim samples have 5.2g and 7.2g mass, respectively. Chirp base excitation with chirp acceleration amplitudes ranging from $0.8 \mathrm{~g}_{0}$ to $2.4 \mathrm{~g}_{0}$ was applied.

Fig. 13 illustrates the voltage variation due to an added tip mass for the No-Shim and Alu-Shim samples, using a $0.88 \mathrm{~g}_{0}$ chirp amplitude. In both samples, the maximum voltage output is increased 
1 by adding the tip mass. The added tip mass creates a significant transient in the voltage variation, 2 which can be easily seen for both cases in Fig. 13 (a) and (b). After approximately $0.09 \mathrm{~s}$ in the Alu3 Shim sample, this transient vanishes but decays less rapidly in the Alu-Shim sample. A reason for 4 this can be the greater stiffness contributed by the additional layers in the Alu-Shim sample.

(a)

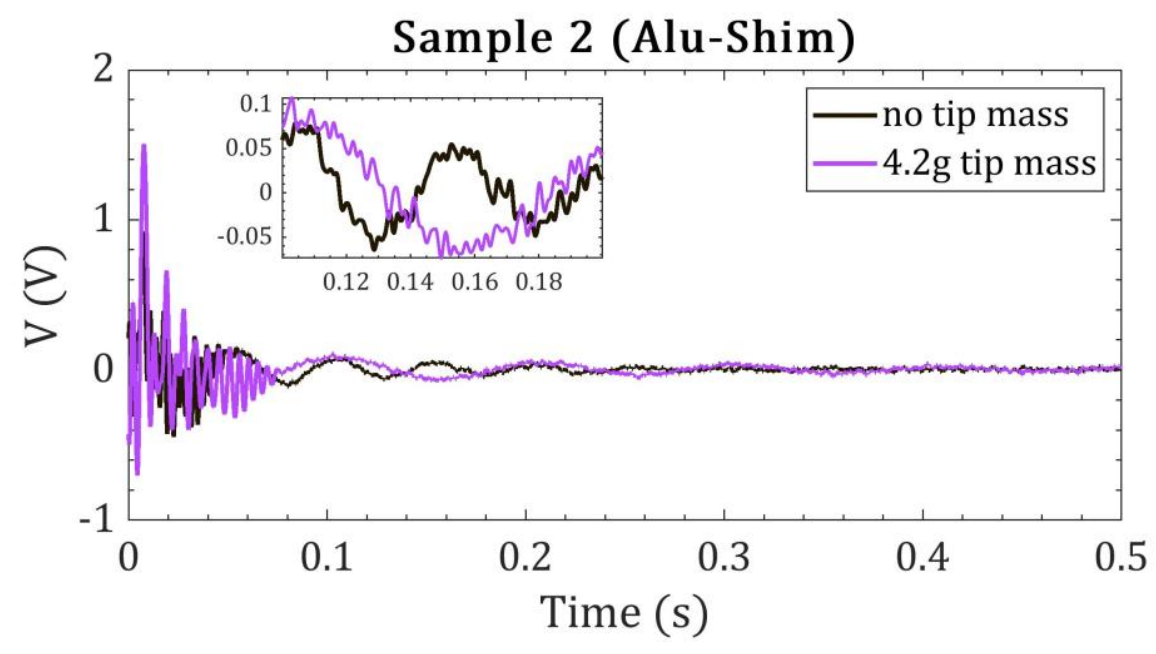

(b)

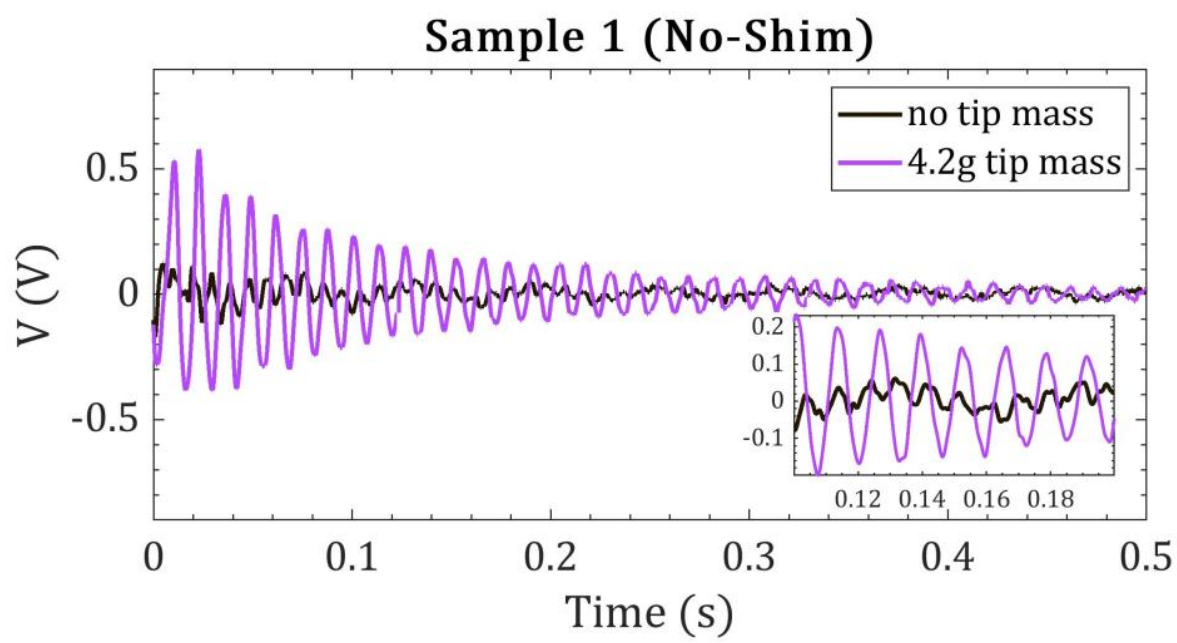

5 Fig. 13. Voltage output for samples with and without tip-mass and $A_{c} / g_{0}=0.88$ (a) Alu-Shim and (b) No-Shim.

Fig. 14 compares the maximum voltage measured in the Alu-Shim and No-Shim samples with and without added tip mass at various $A_{c}$ values. Greater chirp excitation generally causes a greater voltage. As expected from the state-of-art [49], the maximum voltage is increased by adding tip mass.

11 the fractional voltage increase is greater for the No-Shim sample. This is partly because of a greater 12 ratio of the added tip mass to the total harvester mass for the No-Shim sample. Another aspect is that 13 the No-Shim sample is less stiff. The average generated power is of significance from an energy 
1 harvesting perspective. The root mean square (RMS) generated power during the first $1 \mathrm{~s}$ after chirp

2 excitation is shown in Fig. 15 for all the configurations tested. The RMS power for the No-Shim 3 sample is significantly increased by adding the tip mass at all $A_{c}$ values. However, adding tip mass 4 to the Alu-Shim sample did not change the RMS power significantly unless $A_{c}>1.8 \mathrm{~g}_{0}$.

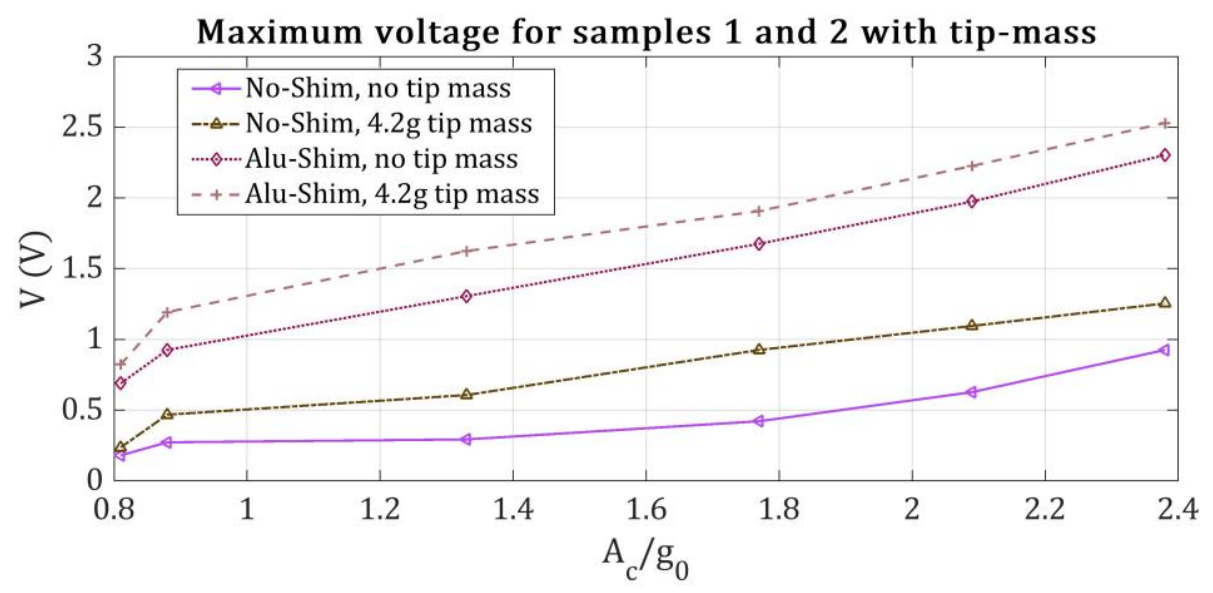

Fig. 14. Maximum voltage for Alu-Shim and No-Shim samples against excitation chirp amplitude.

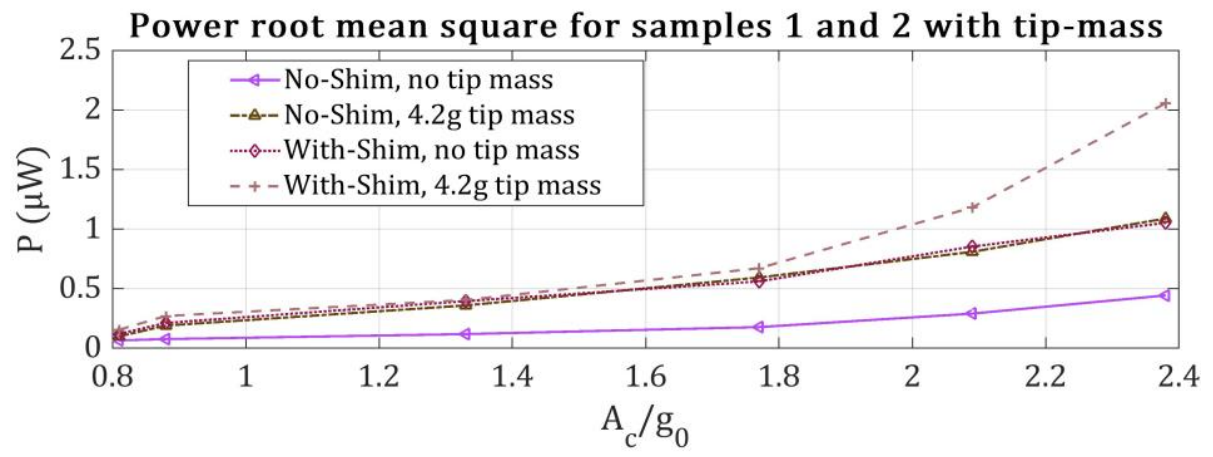

Fig. 15. Root mean square power for Alu-Shim and No-Shim samples against excitation chirp amplitude.

As a preliminary illustration of the tip mass effect on the resonant behavior, the FFT is applied to the harvester voltage signals of Fig. 13, and the results are shown in Fig. 16. The FFT is shown in the frequency range of $0-120 \mathrm{~Hz}$ for clarity. While two resonances are clearly visible for the No-Shim samples, only one resonance can be seen for each Alu-Shim sample. In both sample types, adding tip mass decreases the first mode resonant frequency. However, the resonant peak value in the Alu-Shim sample remains almost constant with added tip mass while the peak value is sharply reduced for the No-Shim sample. Notice that the second-mode resonant peak increases considerably due to the tip mass for the No-Shim sample, indicating the effect of high-frequency harmonics induced by the tip 
1 mass. The appearance of these higher harmonics was also evident in the voltage-time signal of the

2 No-Shim sample in Fig. 13 (b). In order to check the consistency of these trends across a range of 3 excitation amplitudes, the resonant peak values are shown in Fig. 17 as a function of chirp acceleration

4 amplitude $A_{c}$. This shows that the patterns of behavior observed in Fig. 16 remain valid for all the 5 excitation amplitudes.

(a)

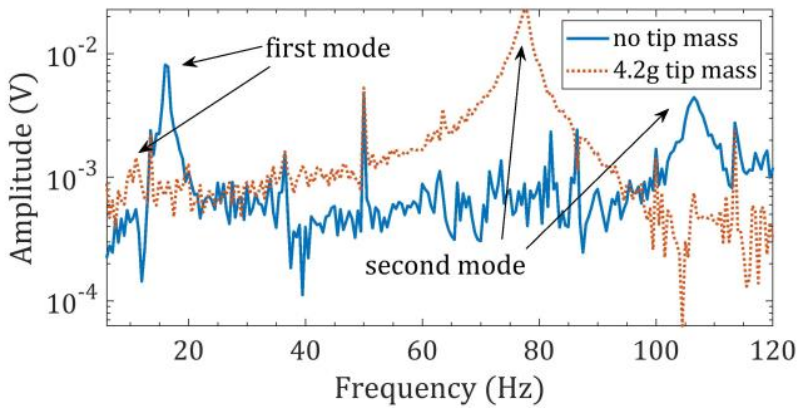

(b)

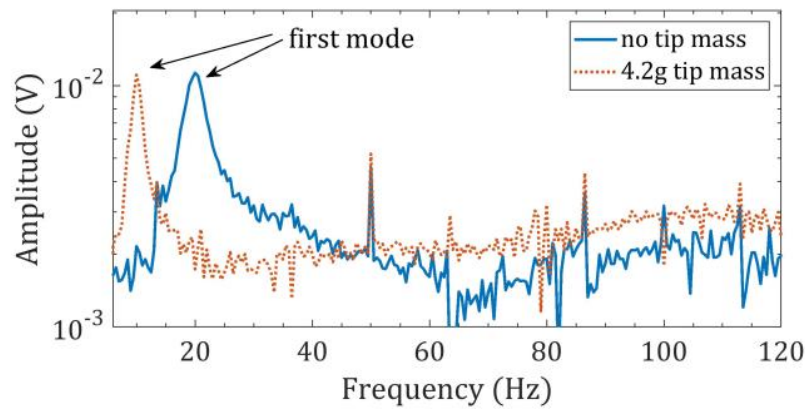

(a)

First mode peak value for No-Shim sample

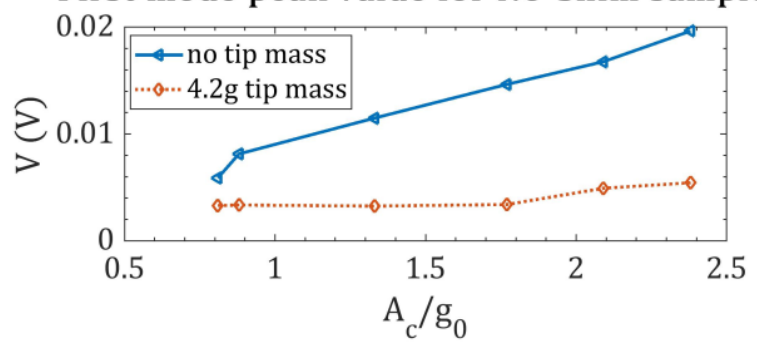

(b)

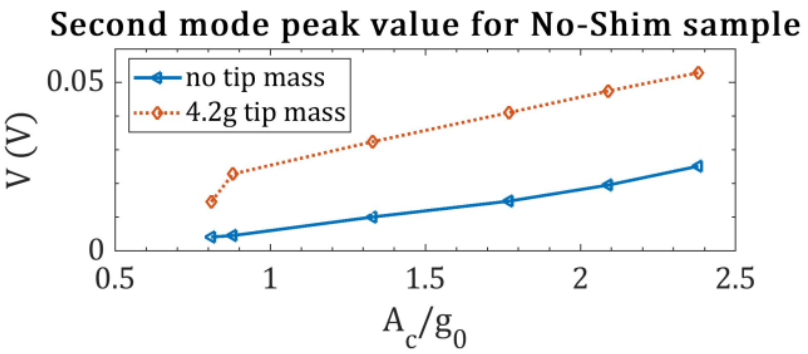

8 Fig. 17. Variation of resonant peak values for sample 1 (No-Shim) due to the added tip mass, (a) the

2 (Alu-Shim) at $0.88 \mathrm{~g}_{0}$ base chirp excitation.

$$
\text { first mode and (b) the second mode }
$$

The averaged resonant frequencies, extracted using the STFR process, are shown in Table 2 for both No-Shim and Alu-Shim samples with and without tip mass when the $0.88 \mathrm{~g}_{0}$ chirp amplitude was applied. For both samples, added tip mass reduces resonant frequencies, as expected.

Table 2. Resonant frequencies for sample 1 (No-Shim) and sample 2 (Alu-Shim) with and without tip mass.

\begin{tabular}{ll} 
Description & $\bar{\omega}_{d}(\mathrm{~Hz})$ \\
\cline { 2 - 3 } & no tip mass $4.2 \mathrm{~g}$ tip mass
\end{tabular}




\begin{tabular}{lccc}
\hline No-Shim sample & & & \\
- first mode & $16.2 \pm 0.4$ & $8.2 \pm 0.2$ & 0.51 \\
- second mode & $108.6 \pm 3.5$ & $78.0 \pm 3.6$ & 0.72 \\
Alu-Shim sample & & & \\
- first mode & $21.3 \pm 0.7$ & $10.8 \pm 0.5$ & 0.51 \\
\hline
\end{tabular}

Fig. 18 shows how the damping coefficients, extracted by the STFR method, vary with chirp 3 amplitude and added tip mass for both samples. In all cases, the damping coefficient shows an 4 increasing trend with chirp excitation amplitude. Added tip mass increases the tip deflection, and

(a)

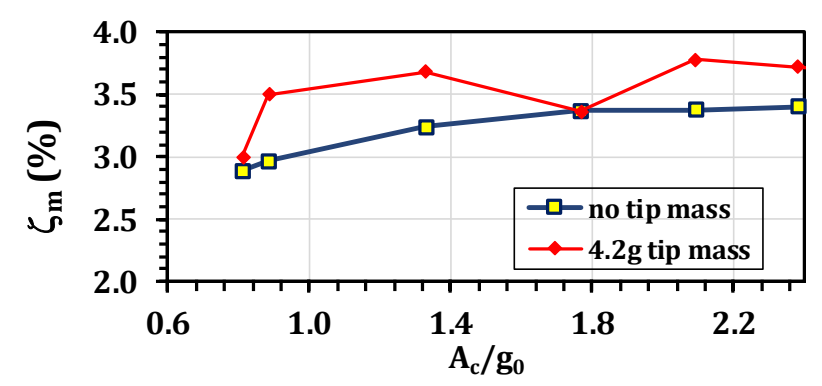

(b)

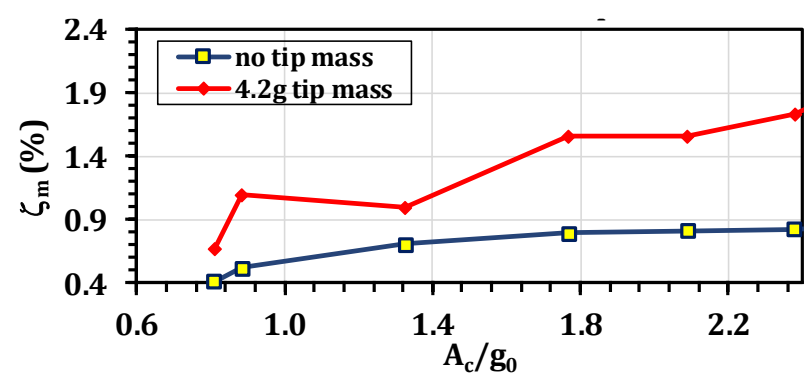

11 Fig. 18. Damping coefficient variations of first-mode due to adding $4.2 \mathrm{~g}$ tip mass for (a) sample 1 (No-Shim) and (b) sample 2 (Alu-Shim).

\subsection{Group II tests (the brass-substrate MFC with and without adhesive tape)}

Group II tests are designed to clarify the structural damping effect of adhesive layers. As in Crandall's study [48], by adding an adhesive damping tape to the beam, the adhesive tape's structural damping can be clarified. The adhesive tape is a strip of Tesa ${ }^{\circledR}$ rubber-type adhesive tape with the dimensions $60 \times 20 \times 1.2 \mathrm{~mm}^{3}$. Two samples in this group are tested; sample 3 , which does not have the adhesive damping tape, and sample 4, to which the adhesive damping tape is attached. The substrate for both samples is a $100 \mu \mathrm{m}$ brass shim. The samples are also tested with an added tip mass of $9.2 \mathrm{~g}$. 
$1 \quad$ Fig. 19 illustrates the time signals for samples 3 and 4 with and without the 9.2g tip mass. The

$29.2 \mathrm{~g}$ added tip mass adversely affects the harvester voltage generation performance reducing the peak

3 voltage magnitude. Regardless of the tip mass, when the adhesive tape is added to the substrate, the

4 output voltage is decreased. The tests are repeated for different excitation amplitudes, and the peak

5 voltage is shown in Fig. 20 versus the applied force. Fig. 20 shows that adding the adhesive tape

6 reduces the peak voltage for all excitation amplitudes. Exploring the root of these voltage variations

7 is possible by extracting the modal parameters in the different configurations because the

8 piezoelectric layer is identical in all these configurations.

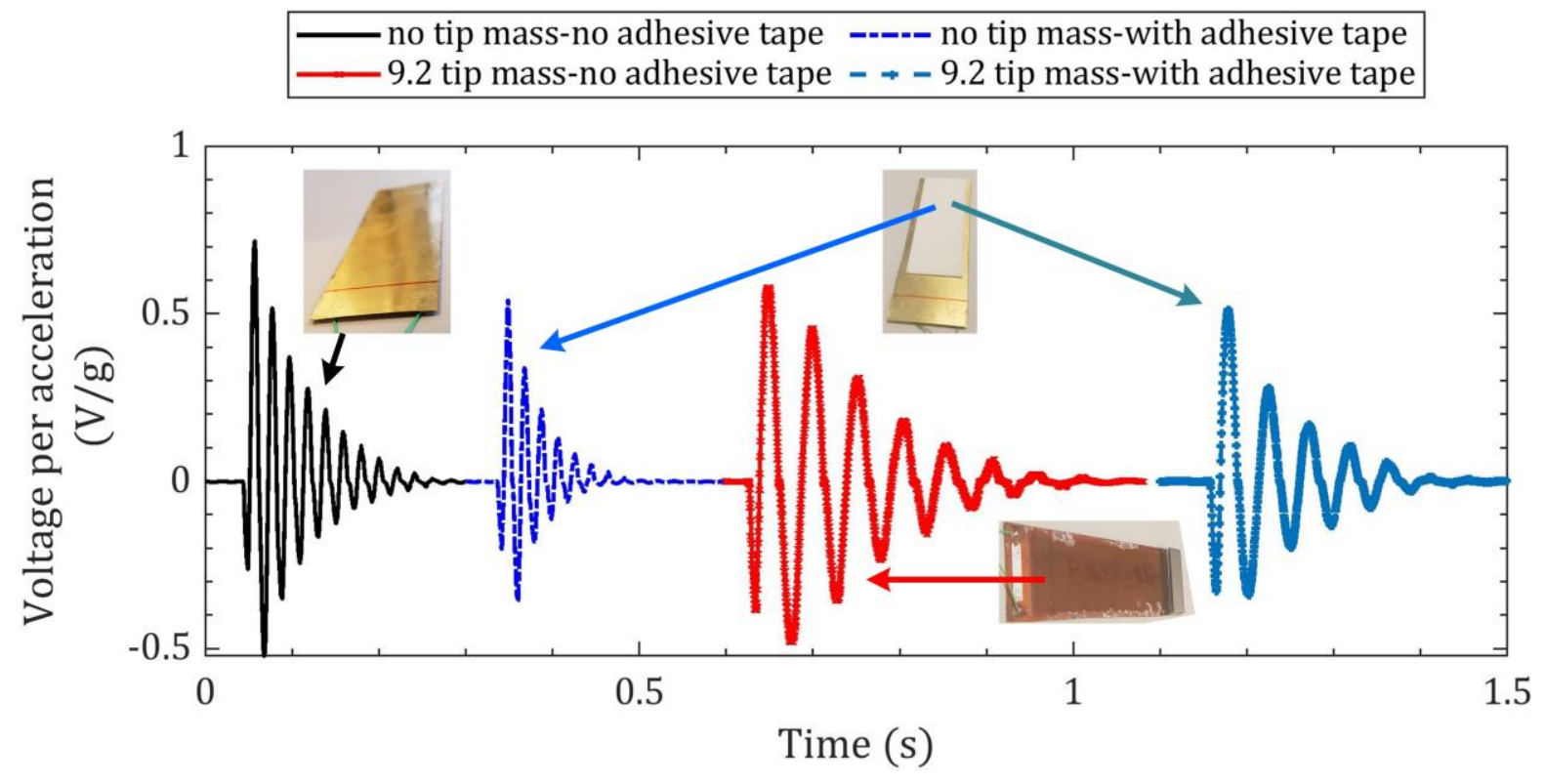

10 Fig. 19. The comparison of time signals for samples 3 (no adhesive tape) and 4 (with adhesive tape) with and without the $9.2 \mathrm{~g}$ tip mass. 


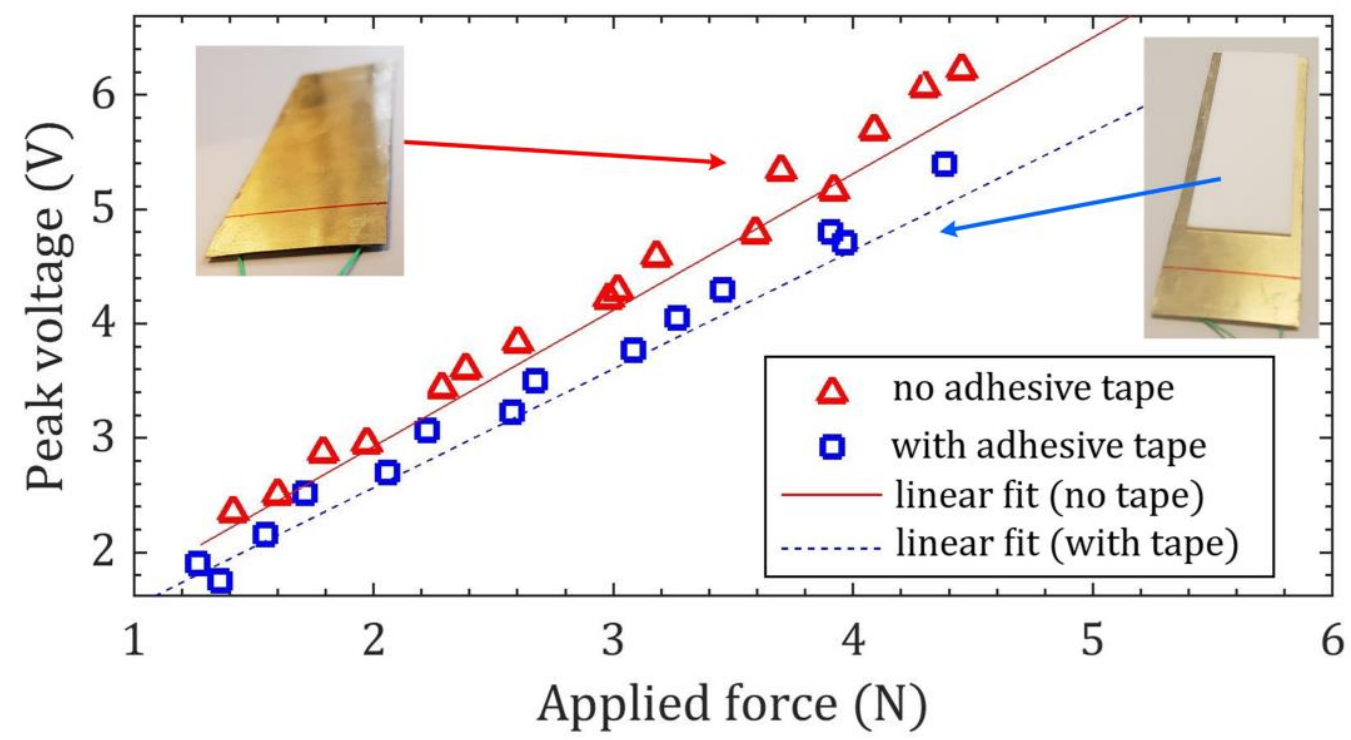

Fig. 20. Peak voltage for samples 3 (no adhesive tape) and 4 (with adhesive tape) versus base excitation amplitude (equivalent to applied force).

Fig. 21 (a) and (b) respectively present the natural frequencies and damping coefficients in the case with adhesive tape and a 9.2g tip mass. As expected, the tip mass decreases the natural frequency, while the adhesive tape has a negligible effect on the natural frequency. Fig. 21 (b) shows that the

7 adhesive tape and the tip-mass increase the damping coefficient. The adhesive tape has a more 8 substantial effect on the damping increase than the $9.2 \mathrm{~g}$ added tip mass, which is a reasonable choice 9 of the tip-mass. These damping increases are consistent with the reduction of peak voltage due to the 10 adhesive tape and the tip mass observed in Fig. 19 and Fig. 20. The adhesive tape's structural damping 11 can be obtained by subtracting the damping coefficient of sample 3 (no adhesive tape) from sample 124 (with adhesive tape).

(a)

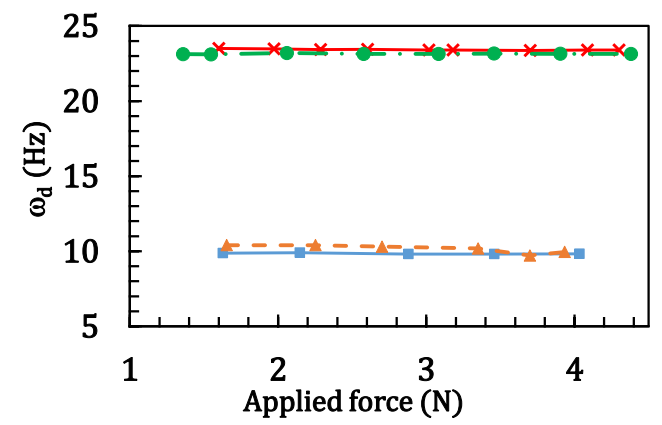

(b)

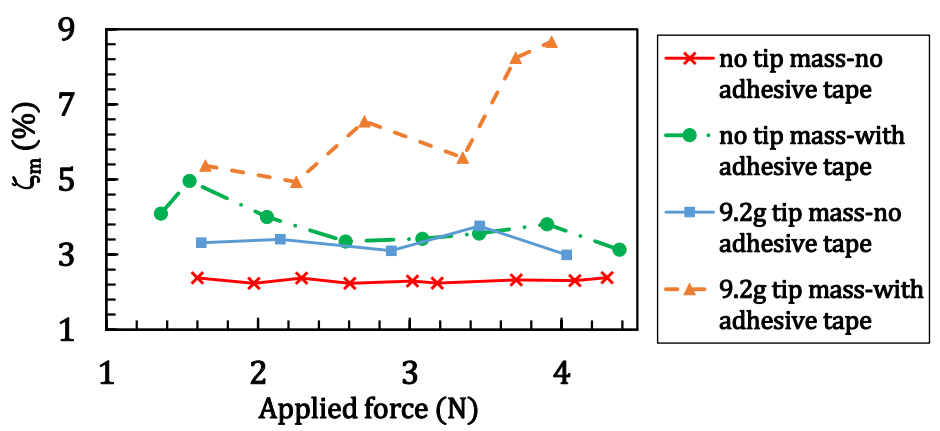

13 Fig. 21. The comparisons of (a) the natural frequency and (b) damping coefficient for samples 3 (no adhesive tape) and sample 4 (with adhesive tape) with and without a 9.2g tip mass. 


\subsection{Group III tests (the copper-substrate MFC with different bonding layers)}

Group III tests investigate the effect of the bonding layer on voltage generation and damping coefficient. Samples 5, 6, and 7 with three different bonding layers, as described in Table 1, are tested in this group. The bonding layer for samples 5 and 6 is made of the same material but with different thicknesses, while the sample 7 bonding layer has the greatest thickness, with an elastic rubber-type material.

Fig. 22 (a) and (b) respectively show the maximum and RMS voltage outputs for different bonding layers. The $1200 \mu \mathrm{m}$ Tesa ${ }^{\circledR}$ bonding layer results in $17 \%$ lower maximum voltage and $32 \%$ lower RMS voltage than that of the $100 \mu \mathrm{m}$ bonding layer. The RMS voltage variations are thus more significant than the maximum voltage variations. To further investigate this, the damping coefficients

11 were calculated in each case.

(a)

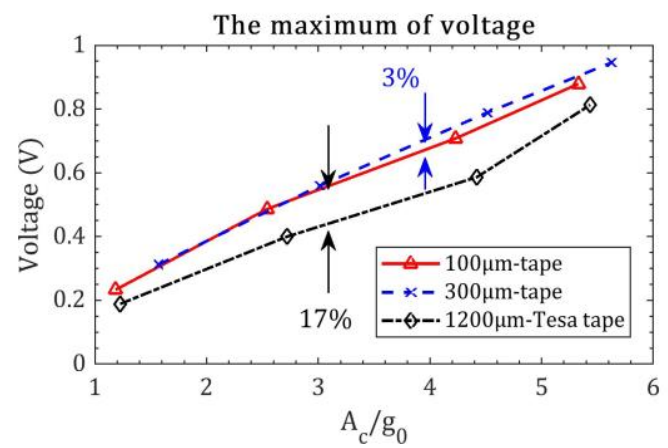

(b)

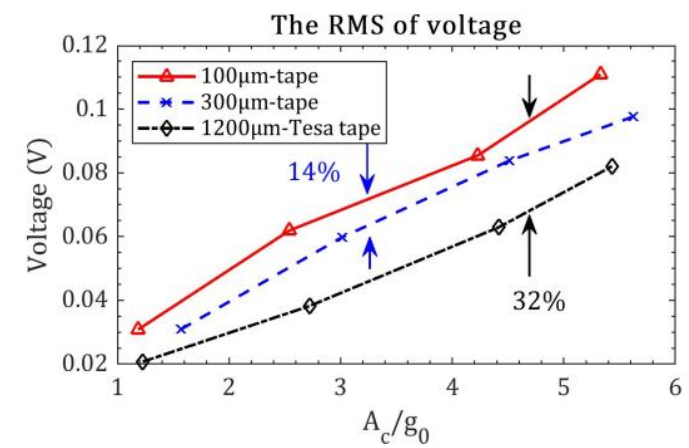

Fig. 22. (a) The maximum and (b) the RMS voltage for three different bonding layers (sample 5, 6, and 7) versus base excitation amplitude.

The identified damping coefficients shown in Fig. 23 indicate that the contact layer substantially influences damping, while no significant natural frequency variations are observed. The $100 \mu \mathrm{m}$ and $300 \mu \mathrm{m}$ double-sided tape bonding layers have the lowest and the highest damping coefficients, while the $1300 \mu \mathrm{m}$ rubber-type bonding layer damping between them. Fig. 23 shows that apart from the bonding layer thickness, the bonding layer damping also depends on the bonding layer material. Khazaee, Rezania, and Rosendahl [21] also demonstrated that the bonding layer state could change the damping coefficient. Therefore, the bonding layer damping is categorized as structural damping in this study, as it depends on the material dimensions and the material type. Table 3 gives the average variation of the damping coefficient due to different bonding layers. The bonding layer damping can vary up to $68 \%$ only due to adding two extra $100 \mu \mathrm{m}$ bonding layers, while the rubber-type tape bonding layer increases the damping by $13 \%$. 
The damping coefficient rise causes the decaying voltage output to decay more rapidly, leaving a smaller RMS voltage. Therefore, the RMS voltage variations can be attributed to increased damping coefficient.

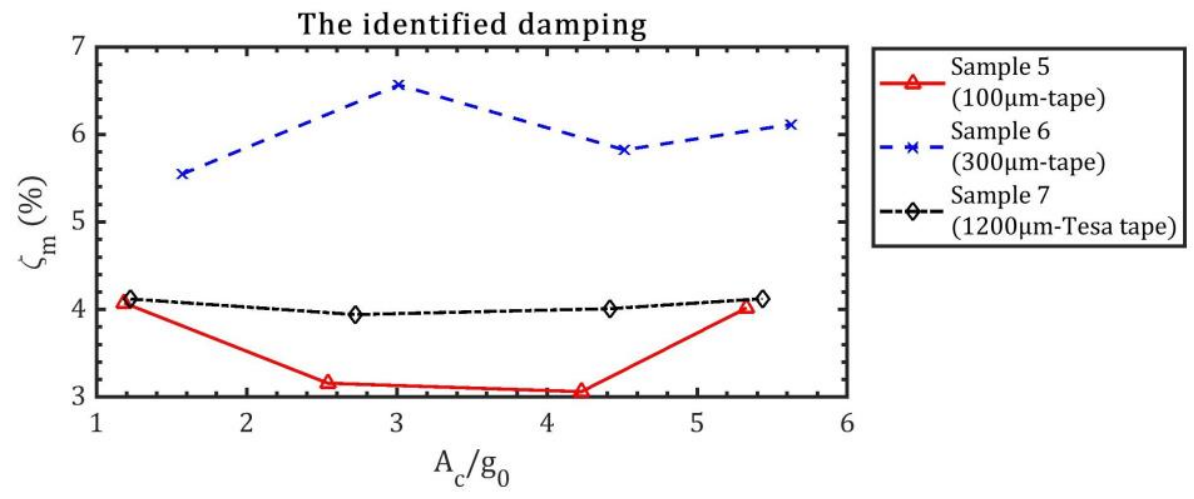

Fig. 23. The bonding layer's effect on the damping for the $100 \mu \mathrm{m}, 300 \mu \mathrm{m}$, and Tesa ${ }^{\circledR}$ tape bonding layers (samples 5, 6, and 7, respectively).

Table 3. The comparison of the damping for the $100 \mu \mathrm{m}, 300 \mu \mathrm{m}$, and Tesa ${ }^{\circledR}$ tape bonding layers (samples 5, 6, and 7)

\begin{tabular}{cccc}
\hline Sample No. & Bonding layer & $\zeta_{\mathrm{m}}(\%)$ & Variation \\
\hline Sample 5 & $100 \mu \mathrm{m}$ tape & 3.6 & - \\
Sample 6 & $300 \mu \mathrm{m}$ tape & 6.0 & $68 \%$ \\
Sample 7 & $1200 \mu \mathrm{m}$ Tesa ${ }^{\circledR}$ tape & 4.1 & $13 \%$ \\
\hline
\end{tabular}

\section{Discussions and application notes}

\section{$11 \quad$ 5.1. The contribution of viscous and structural damping}

Experimental results show that the air damping varies as the base excitation amplitude increases.

13 This subsection gives a detailed summary of the contributions for all the tested samples.

Fig. 24 shows the percentage contribution of viscous air damping to the total damping for the first two bending modes of sample 1 (No-Shim) and the first bending mode of sample 2 (Alu-Shim). The total damping comprises a structural component and a viscous air damping component. Thus the percentage contribution of structural damping is $100 \%$ minus that of viscous air damping. Overall, with increasing chirp acceleration amplitude $\left(A_{c}\right)$, the beam-tip deflection increases, and the percentage contribution of air damping increases accordingly. There is a corresponding reduction in 
1 the percentage contribution of structural damping. Nonetheless, even though the role of structural

2 damping reduces with greater amplitude excitations, more than $80 \%$ of the total damping in the Alu-

3 Shim sample is due to the structural damping. This is because of the bonding layer. By contrast,

4 approximately $70 \%$ of the total damping in the first mode is due to air damping for the No-Shim

5 sample. In the No-Shim sample, air damping dominates when $A_{c} / g_{0}>1.3$. This highlights the

6 necessity of accounting for both structural and air damping, depending on the design of an energy

7 harvester.

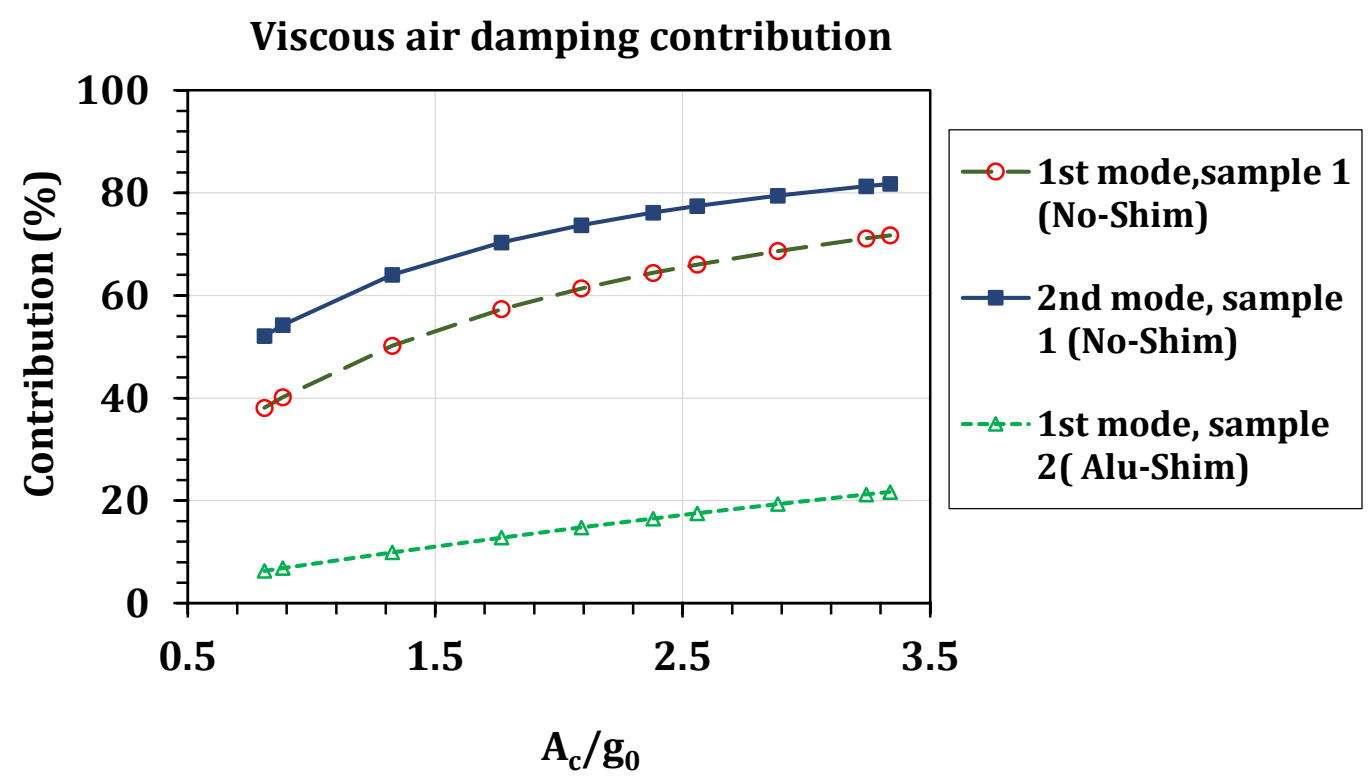

Fig. 24. Percentage contribution of viscous air damping in sample 1 (No-Shim) and sample 2 (AluShim) versus acceleration excitation amplitudes.

The viscous and structural damping contributions are different for different energy harvesters and depend on the bonding layer material, bonding layer thickness, and tip deflection. Table 4 presents the average of viscous and structural damping for all the test samples. Overall, the structural damping contribution is considerable compared to the viscous air damping, which can be approximately $37 \%$ to $85 \%$, depending on the design. The MFC structural damping effect is much smaller than the bonding layer damping effect; for instance, there was only $0.3 \%$ structural damping for the MFC itself, compared with $2.8 \%$ for the epoxy bonding layer. In a typical energy harvester with a substrate shim and an epoxy bonding layer, the structural damping can contribute $75 \%$ or more of the total damping. 
Table 4. The contribution of the viscous and structural damping for all the group tests.

\begin{tabular}{|c|c|c|c|c|c|c|c|}
\hline $\begin{array}{l}\text { Sample } \\
\text { No. }\end{array}$ & Piezoelectric & Substrate & Contact layer & $\begin{array}{c}\text { Total } \\
\text { damping } \\
(\%)\end{array}$ & $\begin{array}{l}\text { Structural } \\
\text { damping } \\
(\%)\end{array}$ & $\begin{array}{c}\text { Viscous } \\
\text { damping } \\
(\%)\end{array}$ & $\begin{array}{c}\text { Structural } \\
\text { damping } \\
\text { contribution } \\
\text { in the total } \\
\text { damping }(\%)\end{array}$ \\
\hline 1 & & - & - & 0.81 & 0.31 & 0.50 & 37.9 \\
\hline 2 & & $\begin{array}{l}120 \mu \mathrm{m} \\
\text { aluminum }\end{array}$ & 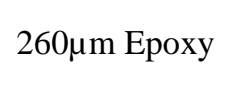 & 3.34 & 2.84 & 0.50 & 85.0 \\
\hline 3 & & $100 \mu \mathrm{m}$ brass & 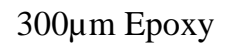 & 2.31 & 2.10 & 0.21 & 91.0 \\
\hline 4 & & $\begin{array}{c}100 \mu \mathrm{m} \\
\text { brass+1 Tesa } \\
\text { adhesive layer }\end{array}$ & 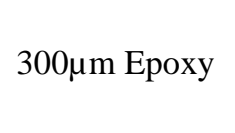 & 4.87 & 3.79 & 1.08 & 77.7 \\
\hline 5 & MFC & $100 \mu \mathrm{m}$ copper & $\begin{array}{c}100 \mu \mathrm{m} 3 \mathrm{M} \\
\text { Company } \\
\text { double-sided } \\
\text { tape }\end{array}$ & 3.58 & 1.22 & 2.36 & 34.0 \\
\hline 6 & & $100 \mu \mathrm{m}$ copper & $\begin{array}{c}300 \mu \mathrm{m} 3 \mathrm{M} \\
\text { Company } \\
\text { double-sided } \\
\text { tape }\end{array}$ & 6.01 & 3.65 & 2.36 & 60.7 \\
\hline 7 & & $100 \mu \mathrm{m}$ copper & $\begin{array}{c}1200 \mu \mathrm{m} \mathrm{Tesa}^{\circledR} \\
\text { double-sided } \\
\text { tape }\end{array}$ & 4.05 & 1.69 & 2.36 & 41.7 \\
\hline
\end{tabular}

\section{$3 \quad$ 5.2. The effect of added tip mass on the viscous damping}

4 As was shown in Fig. 18, the added tip mass on samples 1 and 2 increases the effective damping. 5 The results for samples 3 and 4 in Fig. 21 illustrate that added tip mass again increases the damping.

6 Table 5 shows that when the piezoelectric harvester is more flexible (sample 1 with no substrate) the

7 damping increase due to the tip mass is more significant than for the stiffer harvesters with substrate

8 shim. The increased damping can be attributed to an increase in air damping due to increased vibration

9 amplitude. Thus, in all the harvesters with a substrate, the damping increases due to the added tip 10 mass. Consequently, the addition of a tip mass may not necessarily enhance the piezoelectric power 11 generation performance in shock-type base excitation.

Table 5. The variations of the natural frequency and the damping versus the tip mass.

\begin{tabular}{cccccccccc}
\hline tip mass $(\mathrm{g})$ & \multicolumn{2}{c}{0} & \multicolumn{2}{c}{4.20} & \multicolumn{2}{c}{9.20} & \multicolumn{2}{c}{ Variation (\%) } \\
\hline Sample No. & $\omega_{n}(\mathrm{~Hz})$ & $\zeta_{m}(\%)$ & $\omega_{n}(\mathrm{~Hz})$ & $\zeta_{m}(\%)$ & $\omega_{n}(\mathrm{~Hz})$ & $\zeta_{m}(\%)$ & $\omega_{n}$ & $\zeta_{m}$ \\
1 & 16.20 & 0.78 & 8.20 & 1.59 & - & - & -49.38 & 103.85 \\
\hline
\end{tabular}




\begin{tabular}{ccccccccc}
\hline 2 & 21.30 & 3.34 & 10.80 & 3.59 & - & - & -49.30 & 7.49 \\
3 & 23.42 & 2.31 & - & - & 9.84 & 3.31 & -57.97 & 43.73 \\
4 & 23.14 & 3.79 & - & - & 10.14 & 6.52 & -56.2 & 72.13 \\
\hline
\end{tabular}

To assist understanding of the resonant behavior of the harvester with an added tip mass, a simple 4 two-degree of freedom (2DOF) system is considered, which comprises two masses $M_{1}, M_{2}$, two 5 springs, $k_{1}, k_{2}$, and one damper, $c_{1}$, as shown in Fig. 25 (a). Parameters are chosen for the standard 6 system (System A) without tip-mass to fit the first and second mode resonant frequencies and the 7 damping coefficients observed in the No-Shim sample. In System B, an added tip mass is appended 8 to the free end of the 2DOF system. The damper in System B may be increased by a factor $\alpha$ to 9 represent the observed damping increase due to air damping when a tip mass is added. In Fig. 25 (b), 10 two bending modes in the beam and their analogs in the 2DOF system are shown. In a 3-1 mode 11 piezoelectric harvester covered by electrodes on both sides, the generated voltage is proportional to 12 the integral of normal strain over the length. This, in a bending beam, is proportional to the curvature 13 integral, which implies that the generated voltage is proportional to the cantilever end rotation $\theta$. 14 Hence $V \propto \theta$ as indicated in Fig. 25 (b). In the 2DOF analogue, the equivalent measure is the extension of spring $k_{2}$, given by $x_{2}-x_{1}$.

(a)
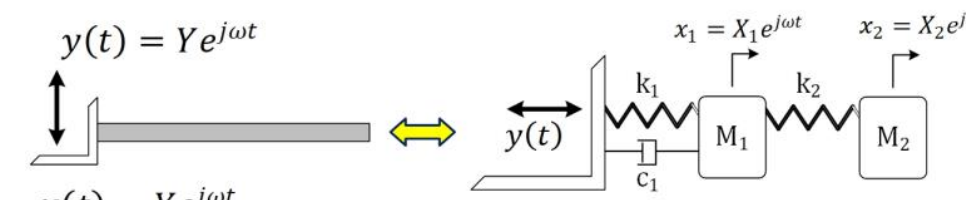
Standard System (System A) $\mathrm{M}_{1}=\mathrm{M}_{2}=\mathrm{m}=2.5 \mathrm{~g}$ $\mathrm{k}_{1}=51.6 \mathrm{~N} / \mathrm{m}$ $\mathrm{k}_{2}=535.1 \mathrm{~N} / \mathrm{m}$

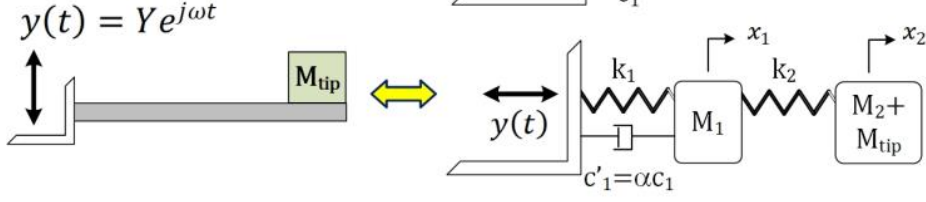
$\mathrm{c}_{1}=0.0041 \mathrm{~N} . \mathrm{s} / \mathrm{m}$

(b)

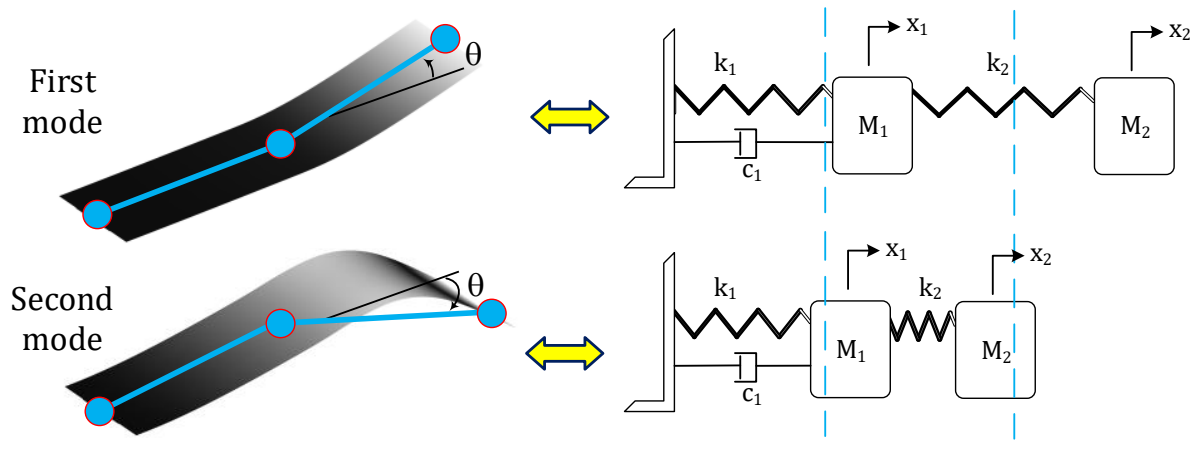


Fig. 25. (a) Representing the harvester transverse-vibration with an analog 2DOF system. (b) The first two bending modes of the cantilever beam and their analog modes in the 2DOF system.

For a given harmonic base excitation amplitude, $Y$, the transmissibility function relating the 4 measured voltage amplitude $V$ to $Y$ is proportional to $\left|\frac{X_{1}}{Y}-\frac{X_{2}}{Y}\right|$, where

$$
\left[\begin{array}{l}
X_{1} / Y \\
X_{2} / Y
\end{array}\right]=\left(\left[\begin{array}{cc}
k_{1}+k_{2} & -k_{2} \\
-k_{2} & k_{2}
\end{array}\right]-\omega^{2}\left[\begin{array}{cc}
m_{1} & 0 \\
0 & m_{2}
\end{array}\right]+j \omega\left[\begin{array}{cc}
c_{1} & 0 \\
0 & 0
\end{array}\right]\right)^{-1}\left(\left[\begin{array}{c}
k_{1} \\
0
\end{array}\right]+j \omega\left[\begin{array}{c}
c_{1} \\
0
\end{array}\right]\right)
$$

Fig. 26 (a) compares $|V / Y|$ for the $4.2 \mathrm{~g}$ added mass with that for no tip mass (System A) for various degrees of air damping increase, $\alpha$. Note that the second-mode resonance peak value increases due to added tip mass for all $\alpha$. On the other hand, depending to the $\alpha$ value, the first-mode resonant peak value may increase or decrease when tip mass is added. Added tip mass increases the tip deflection while greater damping reduces the tip deflection. These two opposing effects balance each-other at a threshold value, $\alpha=\alpha_{\text {thr. }}$. If $\alpha<\alpha_{\text {thr }}$ the effect of added tip mass increases the 11 resonant peak, but when $\alpha>\alpha_{\text {thr }}$ the added tip mass decreases the resonant peak value. The ratio of 12 resonant peak values of the system with tip mass to that without tip mass is shown in Fig. 26 (b), as 13 functions of $\alpha$. The ratio of the second-mode peak values is always greater than unity while the first mode peak value crosses unity when $\alpha \sim 2$. The simple analogue 2DOF system has behavior similar to the experimental results given in Fig. 17. The experimental results show that the first mode peak value is not increased after adding tip mass, whereas the second-mode peak values increased by adding tip mass. It can be concluded that added tip mass, which increases beam tip deflection, also affects the viscous air damping that should be considered in designing an energy harvester. 
(a)

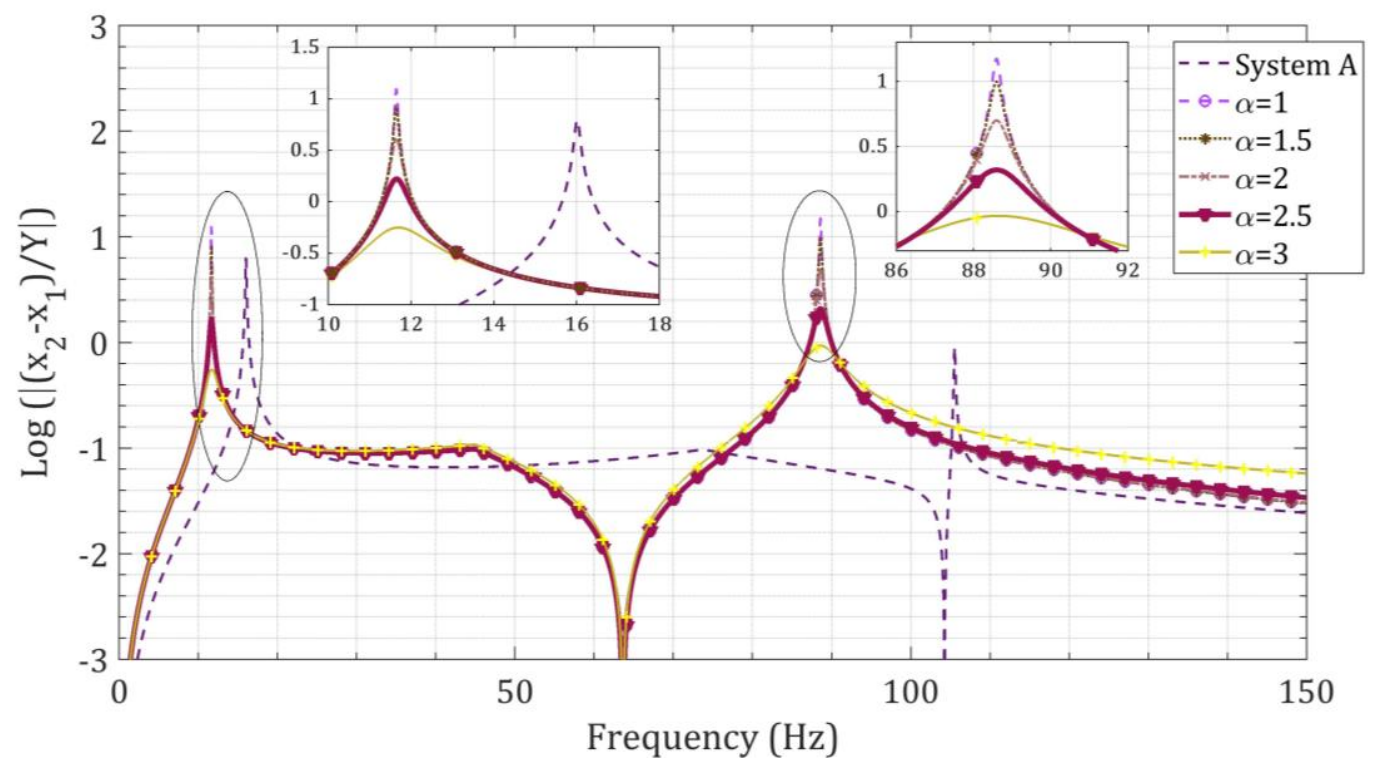

(b)

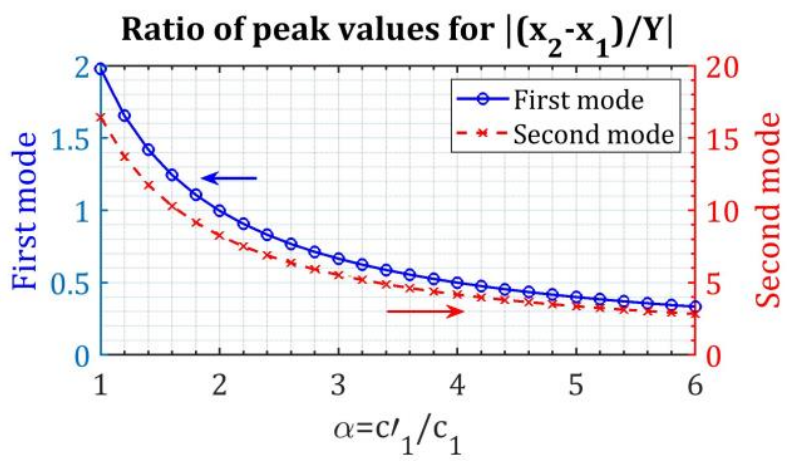

1 Fig. 26. (a) The comparison of $\left|\left(x_{2}-x_{1}\right) / Y\right|$ between no tip mass and with tip mass (system B)

2 with different damping constants and (b) ratios of peak values of the System B to the System A against $\alpha=c^{\prime}{ }_{1} / c_{1}$ ratio.

5 5.3. The role of the bonding layer

6 The bonding layer in the piezoelectric energy harvesters is a thin layer that joins the piezoelectric 7 and supporting structure. In modeling, the bonding layer is often neglected due to its low thickness.

8 Experimental results in group II and III tests showed that the bonding layer has a small effect on the 9 natural frequency, and therefore, from a stiffness perspective, it seems logical to neglect the bonding 10 layer. However, the experimental tests illustrate the substantial damping and power output variations 11 due to different bonding layers. Thus, the bonding layer can affect the piezoelectric power generation 12 performance because of the damping change. From an energy-harvesting perspective, the less the 
1 damping, the higher the power output is. Thus, knowing the bonding layer's damping effect can lead to higher efficient energy harvesters which minimizing structural energy loss.

3 The measured structural damping coefficients for four different bonding layers are shown in Fig. 27, namely 3M Company double-sided tape, Tesa ${ }^{\circledR}$ double-sided tape, Epoxy 3430, and Epoxy rapid 332. Two of these bonding layers are the double-sided tapes, and two others are the cured epoxy bonding layers. The Tesa ${ }^{\circledR}$ double-sided layer is rubber-based and more elastic than the 3M Company double-sided tape. Results show that the rubber-type double-sided tape has a more significant damping effect than the $100 \mu \mathrm{m}$-thickness $3 \mathrm{M}$ Company double-sided tape. Besides, overall, the double-sided tapes have less damping effect than the epoxy bonding layers.

10 Crandall [48] suggested that the structural damping can be due to the transverse heat flow from 11 the regions under compression to the tensile regions and the acoustic radiation from the vibrating 12 beam. Therefore, if a bonding layer has a greater capacity to emit acoustic radiations or creates greater 13 heat flow, the structural damping may be expected to be higher. The experiments also suggest that 14 that thicker bonding layers provide greater capacity to absorb mechanical energy. Thus, the relatively 15 thick epoxy bonding layers have the highest damping coefficient, while the thin $3 \mathrm{M}$ double-sided tape has the lowest damping.

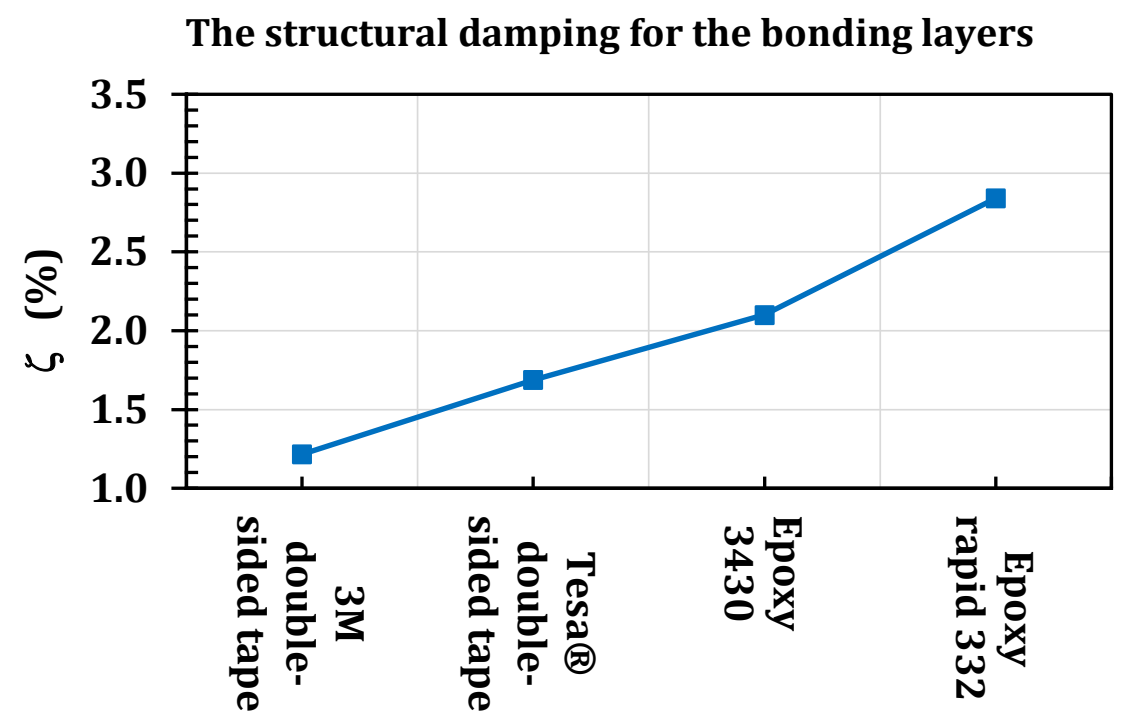

Fig. 27. The structural damping for the different bonding layers (3M Company double-sided tape, Tesa ${ }^{\circledR}$ double-sided tape, Epoxy 3430, and Epoxy rapid 332).

\subsection{The application notes for the design of piezoelectric harvesters}

There is a wide range of vibration sources for the VEH, with different vibration levels and vibration types. The vibration sources for energy harvesting can be continuous, ranging from $0.1 \mathrm{~g}$ to $2 \mathrm{~g}$, or 
1 shock-induced, typically $>1 \mathrm{~g}$. Usually, the shock-induced and impact-based sources have greater

2 peak amplitudes, as their acting time is small, in the order of milliseconds. This study's shock-induced

3 vibrations range from $0.5 \mathrm{~g}_{0}$ to $3.5 \mathrm{~g}_{0}$, a low-to-medium range of accelerations. As in the shock-induced

4 vibrations, the structure vibrates with its fundamental natural frequency; this shock-based beam

5 vibration can be analogous to the resonant excitations; however, the continuous-type acceleration

6 counterpart would be different, depending on the harvester quality factor [50]. This continuous-type

7 acceleration range would be approximately $0.03 \mathrm{~g}_{0}$ to $0.3 \mathrm{~g}_{0}$ at the resonant frequency, which lies

8 within industrial applications such as a water pump [51]. Therefore, the presented experimental

9 results can be beneficial for a low-to-medium range of accelerations in shock-induced and continuous

10 vibration sources.

11 Table 6. Different continuous and shock-induced vibration sources with potential applications for piezoelectric energy harvesting.

\begin{tabular}{cccc}
\hline Vibration source & Vibration type & Peak amplitude $\left(\mathrm{m} / \mathrm{s}^{2}\right)\left[\mathrm{g}_{0}\right]$ & Reference \\
\hline Car engine compartment & Continous & $12\left[1.22 \mathrm{~g}_{0}\right]$ & {$[12]$} \\
Blender casing & Continous & $6.4\left[0.65 \mathrm{~g}_{0}\right]$ & {$[12]$} \\
Clothes dryer & Continous & $3.5\left[0.36 \mathrm{~g}_{0}\right]$ & {$[12]$} \\
Microwave oven top & Continous & $1.11\left[0.11 \mathrm{~g}_{0}\right]$ & {$[45]$} \\
Microwave oven side & Continous & $4.21\left[0.43 \mathrm{~g}_{0}\right]$ & {$[45]$} \\
Water pump & Continous & $8.11\left[0.83 \mathrm{~g}_{0}\right]$ & {$[51]$} \\
Sheep heart & Shock induced & Peak-to-peak: $17.7\left[1.8 \mathrm{~g}_{0}\right]$ & {$[50]$} \\
Car tire & Shock induced & Peak-to-peak: $1570\left[160 \mathrm{~g}_{0}\right]$ & {$[52]$} \\
\hline
\end{tabular}

A typical PEH contains two piezoelectric layers attached to a substrate [49] known as bimorph as it generates higher power output in almost the same volume as a one layer piezoelectric beam. In these typical PEHs, bonding layers are an essential part of the structure. Since the piezoelectric harvester is undergoing dynamic motion for energy harvesting applications, two damping energy dissipation sets occur. The kinetic motion of the structure creates air friction, and the material internal friction creates structural damping. The combined viscous and structural damping model presented here can benefit the study of all types of piezoelectric beams. The results suggest a structural damping contribution of about $40 \%$ in the $100 \mu \mathrm{m}$ bonding layer and $60 \%$ in the $300 \mu \mathrm{m}$ bonding layer.

The average power generation is also vital in shock-induced energy harvesters. As the average generated power is adversely affected by the damping, the damping should be minimized. Therefore, 
1 the harvester configuration should be designed for power maximization. The results suggest that the

2 bonding layer should have a low thickness. In addition, the results highlight the importance of

\section{Concluding remarks}

In this paper, modal parameters are extracted using the Short-Term Fourier Transform and Resampling method for piezoelectric energy harvesters with clamped-free boundary conditions. The emphasis is on the extraction of damping coefficients, using only the voltage response of the harvester to a shock base-excitation. The contributions of structural damping and viscous damping to the total damping are identified. This enables the damping effects of a bonding layer and shim to be interpreted, as well as providing an interpretation of the indirect effects of tip mass on damping. The method further enables the modal parameters to be separately extracted for each mode of vibration. Overall, the Short-Term Fourier Transform and Resampling method provides a promising route for assessing modal parameters, especially damping, in energy harvesters. The results demonstrate the prominent effect of a bonding layer on the structural damping but also indicate the need to include the more subtle effects of air damping when designing energy harvesters. Investigating different bonding layers shows that the bonding layer material and thickness significantly affect the structural damping. An important aspect is that the viscous air damping is not constant with respect to input excitation acceleration, becoming more influential at higher excitation (and hence higher power) levels. Adding tip mass also increases the viscous damping effect due to increasing the tip deflections. For energy harvesting applications, the voltage peak and root mean square will reduce in shockinduced excitations when the damping increases; therefore, the piezoelectric harvester performance will be reduced. The results provide essential guidance for energy harvester design.

\section{References}

[1] Sezer N, Koç M. A comprehensive review on the state-of-the-art of piezoelectric energy harvesting. Nano Energy 2021;80:105567. https://doi.org/10.1016/j.nanoen.2020.105567.

[2] Zou H-X, Zhao L-C, Gao Q-H, Zuo L, Liu F-R, Tan T, et al. Mechanical modulations for enhancing energy harvesting_ Principles, methods and applications _ Elsevier Enhanced 
[3] Sarker MR, Julai S, Sabri MFM, Said SM, Islam MM, Tahir M. Review of piezoelectric energy

Reader.pdf. Appl Energy 2019;255:113871. harvesting system and application of optimization techniques to enhance the performance of the harvesting system. Sensors Actuators, A Phys 2019;300:111634. https://doi.org/10.1016/j.sna.2019.111634.

[4] Kentaro Nakamura. Ultrasonic transducers: Materials and design for sensors, actuators and medical applications. Woodhead Publishing Limited; 2012.

[5] Anton SR, Sodano HA. A review of power harvesting using piezoelectric materials (20032006). Smart Mater Struct 2007;16:R1-21. https://doi.org/10.1088/0964-1726/16/3/R01.

[6] Khazaee M, Rezaniakolaie A, Moosavian A, Rosendahl L. A novel method for autonomous remote condition monitoring of rotating machines using piezoelectric energy harvesting approach. Sensors Actuators A Phys 2019;295:37-50. https://doi.org/10.1016/j.sna.2019.05.016.

[7] Guo L, Lu Q. Numerical analysis of a new piezoelectric-based energy harvesting pavement system: Lessons from laboratory-based and field-based simulations. Appl Energy 2019;235:963-77. https://doi.org/10.1016/j.apenergy.2018.11.037.

[8] Khazaee M, Rezaniakolaei A, Rosendahl LA. A study on interaction effects of different harmonics in translational base excitation for piezoelectric vibration energy harvesters. ECCOMAS Smart 2019, 2019.

[9] Yi JW, Shih WY, Shih WH. Effect of length, width, and mode on the mass detection sensitivity of piezoelectric unimorph cantilevers. J Appl Phys 2002;91:1680-6. https://doi.org/10.1063/1.1427403.

[10] Kim M, Hoegen M, Dugundji J, Wardle BL. Modeling and experimental verification of proof mass effects on vibration energy harvester performance. Smart Mater Struct 2010;19:045023. https://doi.org/10.1088/0964-1726/19/4/045023.

[11] Lallart M, Zhou S, Yang Z, Yan L, Li K, Chen Y. Coupling mechanical and electrical nonlinearities: The effect of synchronized discharging on tristable energy harvesters. Appl Energy 2020;266. https://doi.org/10.1016/j.apenergy.2020.114516.

[12] Roundy S, Wright PK, Rabaey J. A study of low level vibrations as a power source for wireless sensor nodes. Comput Commun 2003;26:1131-44. https://doi.org/10.1016/S01403664(02)00248-7.

[13] Khalili M, Biten AB, Vishwakarma G, Ahmed S, Papagiannakis AT. Electromechanical characterization of a piezoelectric energy harvester. Appl Energy 2019;253. 
[14] Wang X. A study of harvested power and energy harvesting efficiency using frequency

https://doi.org/10.1016/j.apenergy.2019.113585. response analyses of power variables. Mech Syst Signal Process 2019;133:106277. https://doi.org/10.1016/j.ymssp.2019.106277.

[15] DuToit NE, Wardle BL. Experimental verification of models for microfabricated piezoelectric vibration energy harvesters. AIAA J 2007;45:1126-37. https://doi.org/10.2514/1.25047.

[16] Erturk A, Inman DJ. An experimentally validated bimorph cantilever model for piezoelectric energy harvesting from base excitations. Smart Mater Struct 2009;18:025009. https://doi.org/10.1088/0964-1726/18/2/025009.

[17] Kim M, Dugundji J, Wardle BL. Efficiency of piezoelectric mechanical vibration energy harvesting. Smart Mater Struct 2015;24:055006. https://doi.org/10.1088/0964$1726 / 24 / 5 / 055006$.

[18] Hosaka H, Itao K, Kuruda S. Damping characteristics of beam-shaped micro-oscillators. Sensors Actuators, A Phys 1995;49:87-95. https://doi.org/10.1016/0924-4247(95)01003-J.

[19] Sessner V, Liebig W V., Weidenmann KA. Modal damping behavior of plane and 3D curved constrained layer damping CFRP-elastomer-metal laminates. Compos Part C Open Access 2020;2:100037. https://doi.org/10.1016/j.jcomc.2020.100037.

[20] Zhou XQ, Yu DY, Shao XY, Zhang SQ, Wang S. Research and applications of viscoelastic vibration damping materials: A review. Compos Struct 2016;136:460-80. https://doi.org/10.1016/j.compstruct.2015.10.014.

[21] Khazaee M, Rezania A, Rosendahl L. Effect of damage and support damping mechanisms on unimorph piezoelectric energy harvester. J Vib Control 2019:107754631985516. https://doi.org/10.1177/1077546319855162.

[22] Arafa M, Baz A. Energy dissipation characteristics of active pieozoelectric damping composites. Compos Sci Technol 2000;60:2759-68.

[23] Kwak W. Optimal design and experimental verification of piezoelectric energy harvester with fractal structure. Appl Energy 2021;282:116121.

[24] Moradi-Dastjerdi R, Radhi A, Behdinan K. Damped dynamic behavior of an advanced piezoelectric sandwich plate. Compos Struct 2020;243:112243. https://doi.org/10.1016/j.compstruct.2020.112243.

[25] Khazaee M, Rezaniakolaei A, Rosendahl L. A proorf for looking differently into damping modeling in piezoelectric energy harvesting systems. Proc 26th Int Congr Sound Vib ICSV 2019 2019:1-8. 
[26] Cooley CG, Tran TQ, Chai T. Comparison of viscous and structural damping models for piezoelectric vibration energy harvesters. Mech Syst Signal Process 2018;110:130-8. https://doi.org/10.1016/j.ymssp.2018.03.017.

[27] Iglesias FS, Lopez AF. Rayleigh damping parameters estimation using hammer impact tests. Mech Syst Signal Process 2020;135:106391.

[28] D.J.Ewins. Modal Testing: Theory, Practice and Application. Second. RSP; 2000.

[29] He J, Fu Z-F. Modal analysis. 1st ed. Butterworth-Heinemann; 2001.

[30] Paknejad A, Rahimi G, Farrokhabadi A, Khatibi MM. Analytical solution of piezoelectric energy harvester patch for various thin multilayer composite beams. Compos Struct 2016;154:694-706. https://doi.org/10.1016/j.compstruct.2016.06.074.

[31] Shen D, A. Piezoelectric energy harvesting devices for low frequency vibration applications. Auburn University, 2009.

[32] Khazaee M, Nobari AS, Aliabadi MHF. Experimental Investigation of Delamination Effects on Modal Damping of a CFRP Laminate, Using a Statistical Rationalization Approach. Comput. Exp. Methods Struct., vol. 10, 2018, p. 75-103. https://doi.org/10.1142/9781786344977_0003.

[33] Shariyat M, Jahangiri M. Nonlinear impact and damping investigations of viscoporoelastic functionally graded plates with in-plane diffusion and partial supports. Compos Struct 2020;245:112345. https://doi.org/10.1016/j.compstruct.2020.112345.

[34] Vinogradov AM, Schmidt VH, Tuthill GF, Bohannan GW. Damping and electromechanical energy losses in the piezoelectric polymer PVDF. Mech Mater 2004;36:1007-16.

[35] Banks HT, Inman DJ. On damping mechanisms in beams. J Appl Mech Trans ASME 1991;58:716-23. https://doi.org/10.1115/1.2897253.

[36] Khazaee M, Rezaniakolaei A, Rosendahl L. A comprehensive electromechanically coupled model for non-uniform piezoelectric energy harvesting composite laminates. Mech Syst Signal Process 2020;145:106927.

[37] Ju S, Ji CH. Impact-based piezoelectric vibration energy harvester. Appl Energy 2018;214:139-51. https://doi.org/10.1016/j.apenergy.2018.01.076.

[38] Smart Material Inc.,. April 2020.

[39] Hentschel OP, Scheidt LP Von, Wallaschek J, Denk M. Introduction and evaluation of a damping determination method based on the Short-Term fourier transform and Resampling (STFR). J Theor Appl Mech 2015;53:439-52. https://doi.org/10.15632/jtam-pl.53.2.395.

[40] Khazaee M, Rezania A, Rosendahl L. An experimental study to determine damping of 
piezoelectric harvesters using transient analysis of unified electromechanical voltage equation.

[41] Inman DJ. Engineering vibration. 3rd ed. New Jersey: Prentice-Hall, Inc.; 2001.

[42] Woolam WE. Drag coefficients for flat square plates oscillating normal to their planes in air. Langley Research Center: 1972.

[43] Baker WE, Woolam WE, Young D. Air and internal damping of thin cantilever beams. Int J Mech Sci 1967;9:743-66.

[44] Jacquelin E, Adhikari S, Friswell MI. A piezoelectric device for impact energy harvesting. Smart Mater Struct 2011;20:105008. https://doi.org/10.1088/0964-1726/20/10/105008.

[45] DuToit NE, Wardle BL, Kim SG. Design considerations for MEMS-scale piezoelectric mechanical vibration energy harvesters. Integr Ferroelectr 2005;71:121-60. https://doi.org/10.1080/10584580590964574.

[46] Khazaee M, Rezaniakolaei A, Rosendahl L. An experimental study on macro Piezoceramic fiber composites for energy harvesting. Mater Sci Forum 2019;951:3-8. https://doi.org/https://doi.org/10.4028/www.scientific.net/MSF.951.3.

[47] Khazaee M, Rezaniakolaei A, Rosendahl L. On the effect of driving amplitude, frequency and frequency-amplitude interaction on piezoelectric generated power for MFC unimorph. Proc 26th Int Congr Sound Vib ICSV 2019 2019:1-7.

[48] Crandall SH. The role of damping in vibration theory. J Sound Vib 1970;11:3-18. https://doi.org/10.1016/S0022-460X(70)80105-5.

[49] Li H, Tian C, Deng ZD. Energy harvesting from low frequency applications using piezoelectric materials. 2014. https://doi.org/10.1063/1.4900845.

[50] Jackson, Nathan, Oskar Z. Olszewski, Cian O'Murchu and AM. shock-induced aluminum nitride piezoelectric energy harvester leadless pacemaker. Sensors Actuators, A Phys 2017;264:212-8.

[51] Moosavian A, Khazaee M, Ahmadi H, Khazaee M, Najafi G. Fault diagnosis and classification of water pump using adaptive neuro-fuzzy inference system based on vibration signals. Struct Heal Monit 2015;14:402-10. https://doi.org/10.1177/1475921715591873.

[52] Elfrink R, Matova S, De Nooijer C, Jambunathan M, Goedbloed M, Van De Molengraft J, et al. Shock induced energy harvesting with a MEMS harvester for automotive applications. Tech Dig - Int Electron Devices Meet IEDM 2011:677-80. https://doi.org/10.1109/IEDM.2011.6131639. 